$$
\begin{gathered}
\text { LANGLEY } \\
\text { GRANT } \\
\text { IN-3Q-CR }
\end{gathered}
$$

DEPARTMENT OF MECHANICAL ENGINEERING AND MECHANICS

COLLEGE OF ENGINEERING AND TECHNOLOGY

OLD DOMINION UNIVERSITY

NORFOLK, VIRGINIA 23529

FINITE ELEMENT METHODOLOGY FOR INTEGRATED

FLOW-THERMAL-STRUCTURAL ANALYSES

By

Earl A. Thornton, Principal Investigator

R. Ramakrishnan, Research Associate

and

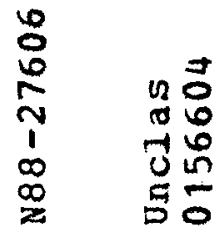

G. R. Vemaganti, Graduate Research Assistant

Progress Report

For the period ended February 29, 1988

Prepared for the

National Aeronautics and Space Administration

Langley Research Center

Hampton, Virginia 23665

Under

Research Grant NSG 1321

Allan R. Wieting, Technical Monitor

LAD-Aerothermal Loads Branch

Submitted by the

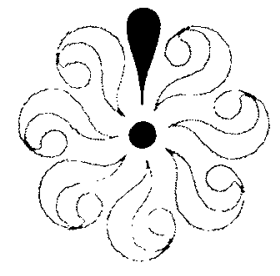

0ld Dominion University Research Foundation

P. 0. Box 6369

Norfolk, Virginia 23508

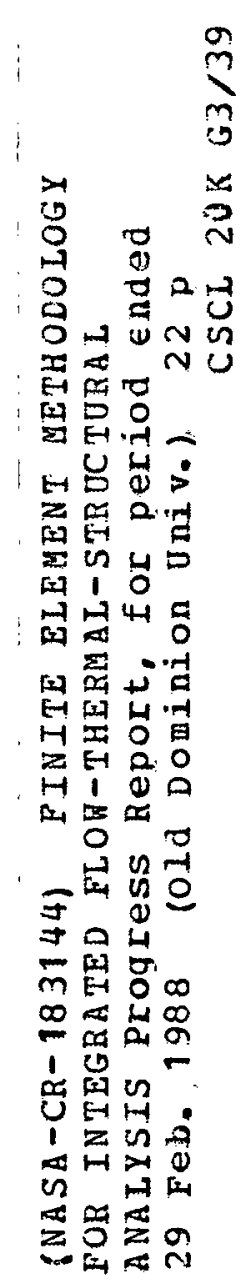

August 1988 


\title{
FINITE ELEMENT METHODOLOGY FOR INTEGRATED FLOW-THERMAL-STRUCTURAL ANALYSES
}

\section{By}

\author{
Earl A. Thornton', R. Ramakrishnan², \\ and 6. R. Vemagant $i^{3}$
}

\section{SUMMARY}

The following papers entitled, "An Adaptive Finite Element Procedure for Compressible Flows and Strong Viscous-Inviscid Interactions" and "An Adaptive Remeshing Method for Finite Element Thermal Analysis," were presented at the June $27-29,1988$, meeting of the AIAA Thermophysics, Plasmadynamics and Lasers Conference, San Antonio, Texas. The papers describe research work supported under NASA/Langley Research Grant NSG 1321, and are submitted in fulfillment of the progress report requirement on the grant, for the period ended February 29, 1988.

1 Professor, Department of Mechanical Engineering and Mechanics, 01d Dominion University, Norfolk, Virginia 23529.

2 Research Associate, Department of Mechanical Engineering and Mechanics, 01d Dominion University, Norfolk, Virginia 23529.

${ }^{3} \mathrm{Gr}$ aduate Research Assistant, Department of Mechanical Engineering and Mechanics, 0ld Dominion University, Norfolk, Virginia 23529. 


\title{
AN ADAPTIVE FWNTE ELEMENT PROCEDURE FOR COMPRESSIBLE FLOWS WTH STRONG VISCOUS-INVISCID INTERACTIONS
}

\author{
R. Ramakrishnan* \\ Earl A. Thornton \\ Old Dominion University \\ Norfolk, Virginia \\ and \\ A.R.Wloting \\ NASA Langley Rosearch Conter \\ Hampton, Virginia
}

ABSTRACT

The use of an adaptive mesh refinement procedure for analyzing high speed compressible flows with strong inviscid-viscous interactions is described. The adaptation procedure which uses both quadrilateral and triangular elements, is implemented with the multistep Galerkin-Runge Kutta scheme. Elements that lie in regions of strong gradients are refined based on inviscid and viscous indicators to obtain better definition of flow features. The effectiveness of the finite element procedure is demonstrated by modelling Mach 11.7 flow over a 15 degree ramp. Numerical results are compared with predictions of the strong interaction theory and experimental data. The influence of factors such as mesh spacing and numerical dissipation on the accuracy of computed results is discussed.

\section{NOMENCLATURE}

$\begin{array}{ll}\text { A } & \text { element area } \\ C & \text { Chapman-Rubesin parameter eq. (33) } \\ C_{1} & \text { skin friction coefficient } \\ C_{H} & \text { heat transfer coefficient, Stanton number } \\ C_{p} & \text { pressure coefficient } \\ C^{1}, \ldots, c^{4} & \text { Runge-Kutta constants } \\ d_{1}, d_{2} & \text { artifical dissipation fluxes } \\ E_{1}, E_{2} & \text { flux components } \\ \text { e } & \text { element error } \\ \text { Fa } & \text { advective flux } \\ \text { FV } & \text { viscous flux } \\ \text { Fh } & \text { heat flux }\end{array}$

\footnotetext{
"Research Associate, Department of Mechanical Engineertng and Mechanics, Member AlAA

- Professor, Department of Mechanical Engineering and Mechanics, Associate Follow ANA

* Head, Aerothermal Loads Branch. Sinuctural Mechanics Division, Member ANA
}

\section{G}

\section{i}

1,12

$M$

[M]

[N]

n

\section{$n_{x}, n_{y}$}

qn

\section{p}

Pr

R

r

Re

S

$T$

$T_{x}, T_{y}$

$t$

$t$

U

$u, v$

$x_{1}, x_{2}$

$\mathbf{Y}$

p

$\alpha$ growth factor, 0q.(35)

unit vector defining velocity change, eq. (7)

components of unit normal vector

Mach number

mass matrix

element interpolation function

unit vector normal to surface

components of unit vector $n$

heat flux normal to surface

pressure

Prandtl number

global nodal residual

coordinate index , eq. (10)

Reynolds number

solution domain boundary

temperature

surface tractions

time

unit vector normal to i

typical conservation variable

velocity components in coordinate directions

coordinate directions

maximum element error

key variable for refinement

threshold value for refinement 
Courant number

$\beta$

threshold value for derefinement, grid stretching parameter

$\begin{array}{ll}\delta_{i j} & \text { Kronecker delta } \\ \gamma & \text { ratio of specific heats } \\ \mu_{2}, \mu_{4} & \text { dissipation constants } \\ \tau_{W} & \text { shearing stress } \\ X & \text { interaction parameter, eq. (33) } \\ \Delta x, \Delta y & \text { grid spacings in coordinate directions }\end{array}$

Subscipts

$\begin{array}{ll}\text { aw } & \text { adiabatic wall } \\ \text { d } & \text { dynamic conditions } \\ i & \text { summation index } \\ j & \text { index for nodes } \\ \text { o } & \text { total conditions } \\ \text { res } & \text { reservoir conditions } \\ \text { w } & \text { wall quantities } \\ \infty & \text { freestream conditions }\end{array}$

\section{Superscripts}

n

index for multistep scheme

time step

\section{INTRODUCTION}

The potential role of computational fluid dynamics (CFD) in the design and analysis of high speed vehicles is being clearly defined by the national aerospace plane, which is envisaged to have applications at flight speeds exceeding Mach 25. Computational techniques that provide good understanding of the flow features and accurate estimates of aerothermal loads is essential in the design of such vehicles since ground based facilities cannot simulate the entire flight envelope. Description of the complex inviscid-viscous interactions with vehicle surfaces is possible with the full Navier-Stokes equations. At the Aerothermal Loads Branch at NASA Langley Research Center, finite element methodology is being developed for integrated fluid-thermal-structural analysis which can accurately predict the heating rates, pressure loads and the themal/structural response.

Compressible flows may contain discontinuities such as shocks as well as regions of high gradients such as boundary layers and shear layers which need to be adequately resolved. Since these flow phenomena occur over small distance scales which are not known apriori, the computational mesh needs to be adapted to model such high gradient regions without the use of an excessive number of elements in low gradient regions. Adaptive mesh refinement procedures can be used effectively to resolve details in the flow region and minimize elements elsewhere. Such procedures lead to highly unstructured meshes. Since finite element methods are characterized by their ease in handling completely unstructured meshes and their ability to include mesh refinement procedures these methods are pursued. Explicit finite element schemes have also demonstrated their capacity to produce good results for a variety of flow situations and configurations $1-4$.

The purpose of this paper is to describe the application of an adaptive refinement procedure to predict viscous compressible flow features. Adaptive mesh refinement procedures for compressible high speed flows are of recent origin. Mesh refinement procedures for triangular finite element meshes were initially detailed by Zienkewicz, Lohner, and Morgan 5 , and the application of these procedures to steady 6 , and transient ${ }^{7}$ compressible flow problems has been demonstrated extensively. Adaptive procedures for finite element meshes with quadrilateral elements have been developed by Oden et. $\left.a\right|^{8}$ and by Shapiro and Murman9. A mesh regeneration scheme developed by Peraire, et. al. 10 has found application in generating meshes comprised of triangles (in 2D) and tetrahedrons (in 3D) for inviscid flows.

Most of the research in adaptive finite element methods has been in inviscid flows. Application of adaptive procedures to model complicated high speed viscous flows are virtually non-existent. A notable exception has been the recent effort to use an upwind finite element formulation 11 and unstructured triangular grids to detail complex flows resulting from shock interaction on a cylindrical leading edge.

The present study extends the adaptive refinement procedure and finite element formulation of reference 12 to predict inviscid and viscous flow details for hypersonic flows with strong inviscid-viscous interactions.

\section{MESH ADAPTATION}

The classical finite element mesh refinement scheme is the addition of elements in regions of high gradients. Elements that lie in these regions are divided into smaller ones by a subdivision process. Both triangular and quadrilateral elements can be enriched by adding a central node and/or midside nodes. Figure 1 (a) illustrates a triangular mesh that has been locally enriched to capture an oblique shock. For a typical quadrilateral element such a subdivision can result in 
the generation of four smaller elements with the possibility of the presence of midside nodes not being connected to neighboring elements. These midside nodes are sometimes called "hanging nodes." Figure 1(b) illustrates a mesh with hanging nodes. The solution algorithm must bo modified to account for hanging nodes, typically by introducing constraint equations. One way to avoid these unconnected nodes is to transition from a crude quad mesh to a fine one using triangular elements. Figure 1(c) shows this type of refinement which needs no special constraint equations and the procedure provides a "natural" way to transition between meshes of different density. Automation of the adaptive procedure is accomplished with refinement indicators.

\section{Refinement Indicators}

The rationale for using refinement indicators is that, while it is possible to predict the location and strength of shocks, boundary layers, etc. for some simple flow situations, the analyst in general will not have prior knowledge of the location of regions containing sharp changes in flow variables. The decision to refine a particular region of the mesh can be based on either a-priori or a-posteriori error estimates. The procedure adopted in this paper is to complete an analysis on a given mesh and then refine the mesh at a certain stage in the analysis. The refinement at this stage is based on inviscid and viscous "error" indicators computed on the initial mesh. The new mesh is used for the subsequent analysis and then further refinements are performed if needed.

The aim of the adaptive mesh refinement is the minimization of maximum errors that occur in the finite element domain. The mesh requirement is then

$$
\operatorname{minimize}(\max \theta i) \quad 1<i<n
$$

where $\mathrm{i}$ denotes the element number, $n$ the number of elements in the domain and $\theta j$ the error in the $i-t h$ element. An optimal mesh then satisfies the equal distribution condition,

$$
\theta_{i}=\text { constant }
$$

which indicates that the error measure is uniformly distributed for all elements of the finite element mesh. The adaptive mesh refinement procedure is designed to add elements in appropriate regions to satisfy Eq. (2). Since the solution is not known a-priori, an approximation to the error measure is computed using the finite element solution. If " $\rho$ " is considered the key variable representative of overall solution behavior, then an error measure for inviscid refinement is given by

$$
\theta_{i}=\sum_{j, k=1,2} \theta_{j k}
$$

where $\theta_{j k}$ are scaled second derivatives with a typical term $\theta_{12}$ defined as

$$
\theta_{12}=\theta_{x y}=\rho_{x y} /\left[\left|\left\{\left|\rho_{x}\right|+\varepsilon \sum_{i=1}^{n d}\left|\rho_{1 x}\right|_{i}\right\}_{y}\right|\right]
$$

Here nd is the number of nodes in element $i$, and the comma implies partial differentiation. The first term in the denominator of equation (4) scales the second derivative of the key variable to ensure that shocks of different strengths are adapted equally. This prevents the strongest shock from being overdefined at the expense of capturing weaker shocks. The coefficient $\varepsilon$ in the second term is used to smooth the indicator in regions of oscillations.

The procedure adopted in this paper is to refine all elements that satisfy the criterion

$$
\theta_{i}>\alpha Y
$$

and derefine all elements that satisfy

$$
\theta_{1}<\beta Y
$$

where $\alpha$ and $\beta$ are preset threshold constants and $Y$ the maximum element error over the entire domain. The key variable used for the inviscid refinement is typically pressure or density and $\alpha$ and $\beta$ are usually 0.2 and 0.1 respectively.

The refinement indicator for viscous regions is based on a variation of the explicit dissipation scheme detailed in reference 13. It uses the notion that near the wall the velocity changes within the boundary layer are maximum in the direction normal to the predominant velocity component. Unit vector $i$ defines the change in the value of the velocity vector $V$ and is given by,

$$
i_{1}=V_{1} / \sqrt{V_{x_{x}^{2}+V_{y}^{2}}}
$$

where $v^{2}=\left(u^{2}+v^{2}\right)$. The expression for element error is given by

$$
\left.\theta_{i}=h^{2} \mid \frac{\partial}{\partial i} \vec{\nabla} \cdot \hat{t}\right) \mid
$$

where unit vector $t$ is defined normal to $i$ and is illustrated in figure 2. As with the inviscid indicator, elements with errors above a preset tolerance value are refined while those with errors below the threshold are derefined.

\section{Adaptive Procedure}

The starting point of the adaptive procedure is a "skeleton" mesh or base mesh which contains only a few elements. The skeleton mesh, which consists of quadrilateral elements, needs to be completely structured to begin the adaptive procedure. A structured mesh implies that each interior node in the domain is surrounded by the same number of elements. Prior to the first analysis, the mesh refinement program does an 
overall refinement where each element of the "skeleton" mesh is subdivided into four quad elements. This procedure is repeated a few times until the nodal density of the resulting mesh is deemed sufficient to ootain a solution which captures the main details of the flow field.

Refinement indicators are computed based on the solution obtained on this "initial" mesh and elements that need to be refined or derefined are identified. All elements in the mesh that have indicators above the preset refinement threshold value are enriched while those elements that have values below the threshold derefinement value are coarsened. The refinement strategy used is such that at each mesh change, only one level of refinement or derefinement is permitted. On refinement of a typical element, the "sub-elements" that result could be all quads, or a combination of quads and triangles. The number and type of the resulting "subelements" depends on the refinement level of elements that surround this element.

Figure 3 shows the elements that result in a typical refinement and coarsening procedure. Figure 3(a) shows the original mesh where elements B, C, and $D$ are to be refined. The mesh that results is seen in Fig. 3(b). If on this refined mesh, element group $C$, which includes "sub-elements" $\mathrm{C} 1, \mathrm{C} 2, \mathrm{C} 3$, and $\mathrm{C4}_{4}$, needs to be coarsened, the mesh that results after derefinement appears in Fig. 3(c).

\section{Boundary Layer Refinement}

In addition to the refinement based on error indicators detailed in earlier sections, the facility to generate layers of structured quad elements at the wall is also included within the framework of the analysis program. This technique is developed to ensure a high density of nodes at the no-slip boundaries to resolve the thin laminar hypersonic boundary layer. The program divides the elements located at the wall into a specified number of sub-elements and elements thus generated can be refined and coarsened at successive refinement levels. The nodal coordinates of these new elements are defined such that the mesh generated is stretched normal to the wall. The location of the nodes along normal lines at each wall location is obtained from the expression of Roberts ${ }^{14}$,

$$
\begin{gathered}
\delta_{i}=\frac{(\beta+1)-(\beta-1) /\left[((\beta+1) /(\beta-1)]^{1-}\right]}{[(\beta+1) /(\beta-1)]^{1-r}+1} \quad 1<\beta<\infty \\
x=x_{w}+\Delta x \delta_{i} \quad j=1, \text { nnew } \\
y=y_{w}+\Delta y \delta_{i}
\end{gathered}
$$

where nnew is the number of new elements that result from the subdivision, $\Delta x$ and $\Delta y$ the grid spacings in the coordinate direction, $\beta$ the grid stretching parameter, and $r$ the coordinate index given by,

$$
r=j \text { /nnew }
$$

where $j$ is the index locating the node from the wall in the normal direction. The nodal unknowns for the new grid points at each wall location is linearty interpolated from the nodal values of the parent element. The meshing procedure clusters more nodes near the wall as $\beta$ approaches unity.

\section{FINTE ELEMENT FORMULATION}

The explicit multistep Galerkin-Runge Kutta formulation used in reference 12 for inviscid flow is extended to model the compressible Navier-Stokes equations. The solution algorithm is applied to the flow equations in conservation form

$$
U_{\imath}+E_{i, i}=0
$$

where $U$ is the vector of variables and $E_{i}$ the flux vectors. The flux vectors can be written as

$$
E_{i}=F_{i}^{a}-F_{i}^{v}-F_{i}^{h}
$$

where Fa , FV and Fh represent the advective, viscous and heat fluxes respectively. The vectors of conservation variables and fluxes are given by

$$
\begin{aligned}
& U=p\left\{\begin{array}{l}
1 \\
u_{1} \\
u_{2} \\
e^{2}
\end{array}\right\} \quad F_{i}^{a}=u_{i} U+p\left\{\begin{array}{l}
0 \\
\delta_{1 i} \\
\delta_{2 i}^{i} \\
u_{i}
\end{array}\right\} \\
& F_{i}^{v}=\left\{\begin{array}{l}
0 \\
\tau_{1 i} \\
\tau_{2 i} \\
\tau_{i j} u_{j}
\end{array}\right\} F_{i}^{h}=\left\{\begin{array}{l}
0 \\
0 \\
0 \\
-q_{i j}
\end{array}\right\}
\end{aligned}
$$

where $\rho$ is the density, $u_{i}$ the velocity components in the coordinate directions, $p$ the thermodynamic pressure, $\theta$ the total energy, $q_{i}$ the heat fluxes, $\tau_{i j}$ the viscous stress components and $\delta_{i j}$ the Kronecker delta. The shear stresses and heat fluxes are given by,

$$
\begin{aligned}
& \tau_{i j}=\lambda u_{k, k} \delta_{i j}+\mu\left(u_{i, j}+u_{i, i}\right) \\
& q_{i}=-k T_{i}
\end{aligned}
$$

where $\mu$ and $\lambda$ are the coefficients of viscosity, $k$ the thermal conductivity and $T$ the temperature. Other constitutive relations employed include:

$$
\begin{aligned}
& e=C_{v} T+\left(u^{2}+v^{2}\right) / 2 \\
& p=\rho R T
\end{aligned}
$$


where $C_{V}$ is the specific heat at constant volume and $R$ the gas constant. The coefficients of viscosity are related by Stokes hypothesis

$$
\lambda=-\frac{2}{3} \mu
$$

and $\mu$ is assumed a function of temperature and obtained by Sutherland's law. The thermal conductivity is computed from the Prandtl number Pr which is assumed constant along with constant specific heats. Equation (11) is solved subject to proper initial and boundary conditions which include: (a) specification of conservation variables on the inflow plane, (b) imposition of no-slip conditions and specified temperatures on aerodynamic surfaces, and (c) outflow surface integrals provided by the finite element formulation.

\section{Galerkin-Runge Kutta Agorithm}

The system of conservation laws shown in Eq. (11) can be written as,

$$
U_{i t}=-E_{i, i}
$$

Application of the method of weighted residuals results in the finite element equations,

$$
\int_{A}[N]\left\{\frac{\partial U}{\partial t}\right\} d A=-\int_{A}[N]\left(E_{i, j}\right) d A
$$

where [N] is the element weighting and interpolation function and $A$ the domain of interest. Integrating by parts on the RHS terms yields.

$$
[M]\left\{\frac{\partial U}{\partial t}\right\}=\int_{A}\{N,\}[N] d A[E\}-\int_{S}\{N\}[N] d S\left[l_{i} E_{i s}\right]
$$

where the fluxes $E_{i}$ are interpolated the same way as the conservation variables.

The time marching scheme is similar to the multistep time integration scheme which appears in reference 9 and can be written as.

$$
\begin{aligned}
& U_{j}^{(k)}=U_{j}^{n}+c^{k}\left[\frac{\Delta t_{i}}{M_{i j}} R_{j}\left(U^{k-1}\right)+D\right] k=1, . .4 \\
& U_{i}^{n+1}=U_{i}^{(4)}
\end{aligned}
$$

where the dissipation operator $D$ can be written as,

$$
D=D_{2}^{n}+D_{4}^{n}
$$

and $\mathrm{D}_{2}$ and $\mathrm{D}_{4}$ are second and fourth difference dissipation terms which may be "frozen" for each timestep.

The second difierence artificial dissipation term is needed to stabilize solutions in the presence of shapp gradients. This dissipation operator is given by,

$$
D_{2}=d_{i, i}
$$

and

$$
d_{i}=K_{i} U_{i}(i \text { not summed })
$$

where $k_{i}$ control the amount of dissipation added and are given by,

$$
k_{i}=\frac{\mu_{2} h^{4}}{4 p}\left|p_{i i i}\right| \text { (i not summed) }
$$

where $\mu_{2}$ is the second difference dissipation constant and $p$ the pressure. The first derivatives of pressure are calculated as element quantities and nodal second derivatives are then obtained from the relation,

$$
\int_{A} N p_{x_{x x}} d A=-\int_{A} p_{y_{x}} N_{x_{x}} d A
$$

where the comma implies differentation.

The linear fourth difference operator is needed to damp out spurious oscillations and provide background dissipation and is given by,

$$
D_{4}=\mu_{4}\left(\Delta x_{i}\right)^{4} U_{\text {'iiii }}
$$

The fourth differences are evaluated by repeated application of a 9-point Laplacian stencil. Typical values of the dissipation constants $\mu_{2}$ and $\mu_{4}$ are $1 / 10$ and $1 / 200$ respectively.

\section{Calculation of Wall Coefficients}

Heat transfer, skin friction and pressure coefficients are essential for design considerations since they provide a direct measure of the severity of aerodynamic loads on flight surfaces. Experimental measurements for hypersonic flow behavior yield surface distributions for pressure loads, heat transfer rates and skin friction coefficients. Accurate computation of the wall coefficients in the finite element methodology is essential since their comparison with experimental data forms the basis for code validation.

The heat transfer and shear stress coefficients are related to the gradients of temperature and fluid velocity at the surface. With a structured computational grid it is possible to calculate derivatives at the wall by simple differencing techniques and obtain results of desired accuracy. For completely unstructured finite 
element meshes the calculation of wall derivatives by differencing is rather complicated. Computation of heat fluxes and shear stresses at the wall is possible in the finite element context by using the "consistent" calculations. These calculations, introduced in reference 13, use equation (19) as the basis for computing wall coefficients.

The finite element equations (eq. 19) can be written as

$$
\frac{\partial U}{\partial t}=\int_{A}\{N, p\}[N] d A\{E\}-\int_{S}\{N\}[N] d S\left[l_{i} E_{i s}\right]
$$

At steady state the transient terms approach zero, implying

$$
\int_{S}\{N\}[N] d S\left[l_{i} E_{i s}\right]=\int_{A}\left\{N_{, i}\right\}[N] d A\left\{E_{p}\right\}
$$

The $x$ and $y$ momentum equations for elements on the surface reduces to,

$$
\begin{aligned}
& \int_{S}\{N\}[N] d S\left\{T_{x}\right\}=\left\{R_{x}\right\} \\
& \int_{S}[N\}[N] d S\left\{T_{y}\right\}=\left\{R_{y}\right\}
\end{aligned}
$$

where $T_{x}$ and $T_{y}$ are the surface tractions and $R_{x}$ and $R_{y}$ the residuals for an element. These equations are assembled for all the elements that lie on the surface. Diagonalizing the matrices defined by the surface integrals in equation (28) yields an explicit procedure for obtaining nodal values for the surface tractions. The surface tractions are related to the stress tensor by the relation,

$$
\left\{\begin{array}{l}
T_{x} \\
T_{y}
\end{array}\right\}=\left[\begin{array}{ll}
\tau_{x x} & \tau_{x y} \\
\tau_{y x} & \tau_{y y}
\end{array}\right]\left\{\begin{array}{l}
n_{x} \\
n_{y}
\end{array}\right\}
$$

where $\tau_{\mathrm{ij}}$ are components of the stress tensor and $n_{x}$ and $n_{y}$ are components of the unit vector $n$, normal to the surface. The wall shearing stress is then given by,

$$
\tau_{w}=T_{y} n_{x}-T_{x} n_{y}
$$

Considering the energy equation for elements that lie on the surface, at steady state the finite element equations can be written as,

$$
\int_{S}\{N\}[N] d S\left\{q_{n}\right\}=\left\{R_{h}\right\}
$$

where $q_{n}$ is the nodal heat flux for an element. Diagonalizing the coefficient matrix and assembling the element equations yields nodal values of the heat flux on the surface. The coefficients of heat transfer $(\mathrm{CH})$, skin friction $\left(C_{f}\right)$, and pressure $\left(C_{p}\right)$ are computed from the relations,

$$
\begin{aligned}
& C_{H}=q_{W} / \rho_{\infty} U_{\infty}\left[\left(\theta+p_{p}+u^{2} /{ }_{2}\right)_{-}-(\theta+p / \rho)_{W}\right] \\
& C_{f}=\tau_{W} / \rho_{\infty} u_{\infty}^{2} \\
& C_{p}=p_{W} /\left(\frac{1}{2} p_{\infty} U_{\infty}^{2}\right)
\end{aligned}
$$

where $p_{\infty}, p_{\infty}$ and $u_{\infty}$ are freestream values and $p_{w}$ is the wall pressure.

\section{COMPUTATIONAL RESULTS}

The ability of the adaptive finite element procedure to predict flow details for viscous flows is illustrated by modelling the hypersonic flow over a ramp. This problem has special interest due to its application in the design of control surfaces for high speed vehicles such as the aerospace plane. Theoretical estimates and experimental results are avaliable to gauge the accuracy of the finite element calculations.

\section{Flow Description}

The problem considered is illustrated schematically in Figure 4. Inflow at Mach 11.68 interacts with the leading edge producing a shock due to the boundary layer displacement effect. The boundary layer does not have enough momentum to overcome the adverse pressure gradient generated by the ramp resulting in flow separation and recirculation near the corner. The separated boundary layer then reattaches downstream of the corner with the surface pressure rising through the separated and reattachment regions. The compression fan generated in the separation zone eventually coalesces downstream to form the induced shock. This shock interacts with the leading edge shock to produce a resultant shock, an expansion fan and a shear layer or slip line.

The interaction between the inner viscous shear layer and the outer inviscid flow can be categorized based on the nature of coupling. At the leading edge of the plate the induced shock and the boundary layer are essentially of the same order and the effects of the interaction can be reasonably predicted by various interaction theories. At the corner the separation results upstream of the induced shock due to the coupling between the shock and the thickening of the boundary layer due to compression. This type of interaction necessitates the need for solution of the full NavierStokes equations.

\section{Strong Interaction Theory}

Near the sharp leading edge the displacement thickness increases from zero and the flow turning outside of the boundary layer causes compression and a leading-edge shock. The strength of the shock depends on the incident Mach number and has a strong effect on the growth of the boundary layer at the leading edge. Very near the leading edge, the pressure at the edge of the boundary layer is very high compared to the freestream pressure marking the region of "strong" 
interaction. Downstream of the leading edge the interaction between the inviscid and viscous flow "weakens" and the wall pressure expands to freestream.

From laminar boundary layer theory the appropriate similarity parameter for the shock-boundary layer interaction is the Lees-Probstein parameter $X$ given by

$$
X=M_{0}^{3} \sqrt{C} / \sqrt{R \theta_{x}}
$$

where $\mathrm{C}$ is the Chapman-Rubesin compressibility parameter, $M_{\infty}$ the freestream Mach number, and $\mathrm{R} e_{x}$ the local Reynolds number. The effects of the coupling between the leading-edge shock and the boundary layer have been predicted by various strong 15,16 and weak 17 interaction theories. The complete interaction theory of Bertram and Blackstock 18 unifies the strong and weak interaction theories by bridging their limits of applicability. The assumptions used in the development of these interaction theories include:

(a) the flow is hypersonic $\left(M_{\infty}>1\right)$

(b) no gas dissociation

(c) Prandtl number constant

(d) vorticity effects due to the curved leading edge shock are negligible, and

$(\theta)$ pressure distibution is assumed to vary exponentially with distance from the leading edge.

The complete theory relates the surface pressure to the interaction parameter $X$ as follows,

$$
\frac{P}{P_{\infty}}=0.83+\frac{3}{4} \sqrt{\frac{\gamma(\gamma+1)}{2}} G X
$$

where $\mathrm{G}$ is the growth factor defined as,

$$
\left.G=1.648 \frac{(\gamma-1)}{2} \frac{T_{w}}{T_{a w}}+0.352\right]
$$

and $\mathrm{T}_{\mathrm{aw}}$ is the adiabatic wall temperature given by

$$
T_{\text {aw }}=T_{\infty}\left[1+\frac{\gamma-1}{2} \sqrt{\operatorname{Pr}} M_{\infty}^{2}\right]
$$

for laminar flows. The variation of heat transfer coefficient with surface temperature in the stronginteraction region is tabulated by $L$ and Nagamatsu 15 . For $\gamma=1.4$, variation of $C_{H}$ with wall temperature $T_{W}$ is given by

$$
C_{H}\left[R e_{X} / X\right]^{1 / 2}=C_{1}
$$

where the constant $C_{1}$ depends on $T_{w}$ and the ratio of specific heats and is obtained from reference 19.

\section{Experimentel Meesurements}

The experimental studies used for validation of the finite element approach were conducted in the Calspan 48-inch shock tunnel. These experiments 20 have generated heat transfer, surface pressure and skin friction measurements for hypersonic conditions ranging from Mach 11.5 to Mach 18.9.

The Calspan shock tunnel consists of a shock tube driver, followed by a conical expansion section and a contoured nozzle to produce uniform axial flow in the test section. The tunnel is started by rupturing a double diaphragm which causes expansion of the air in the high pressure driver section. A normal shock is generated with the high temperature and high pressure air between the normal shock and the driver-driven interface. When the shock front strikes the end of the driven section it leaves a region of stagnant high pressure air. This air is then expanded through a contoured nozzle to desired freestream conditions.

Skin friction transducers, thin-film resistance thermometers and pressure transducers mounted at suitable locations obtain skin friction, heat transfer, and surface pressure measurements. Details of the experimental setup are found in reference 20.

The flow parameters used in the numerical computations correspond to the nominal test conditions for hypersonic flow over a ramp presented in reference 20 and given below

$$
\begin{aligned}
& \text { Mos }=11.68 \\
& R_{\theta_{\infty}}=1.714 E+05 / f t \\
& T_{r e s}=2989 \mathrm{R} \\
& P_{d}=0.3589 \text { psia }
\end{aligned}
$$

The experimental data to be used for comparisons will consist of measurements obtained for two sets of tunnel runs 21 . These measurements correspond to runs 19 and 21 , and flow conditions for these runs are as follows

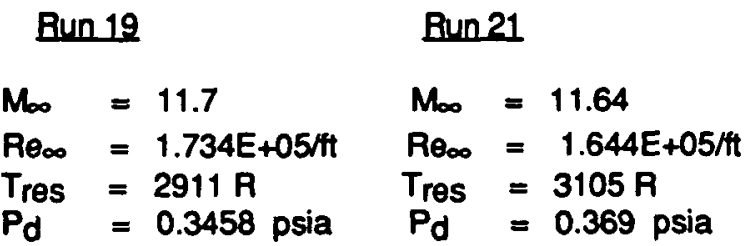

The measurements of runs 19 and 21 yield surface heating rates, pressures and skin-friction. The coefficients of pressure and skin-friction are obtained by dividing experimental values by the freestream dynamic pressure. The coefficient of heat-transfer is obtained from the heat flux at the wall by the relation.

$$
C_{H}=\frac{q_{w}}{\rho_{\infty} u_{\infty} \gamma C_{V}\left[T_{\text {res }}-T_{w}\right]}
$$

where $T_{w}$, the wall temperature, assumed to be the same for both runs, is obtained from reference 20 and the specific heat is assumed constant. 


\section{Computational Strategy}

The hypersonic flow over a ramp results in inviscid-viscous interactions that have distinctly different character in various regions of the domain. Near the leading edge, the mesh needs to be well refined and uniform to capture the gradients in both the streamwise and normal directions. Away from the leading edge, the flow is basically the development of the viscous boundary layer and mesh spacings at the surface in the normal direction are of concern. At the corner, the coupling between the inviscid and viscous regions necessitate the need for adequate refinement to capture the separated and attached regions of flow.

The adaptive finite element approach was implemented on the NAS Cray-2 supercomputer. The analysis is started on an initial mesh and iterations are performed until the $L_{2}$ norm of the residuals of the conservation variables dropped over three orders of magnitude. The mesh is then refined/coarsened based on the inviscid and viscous error indicators. On the final mesh, convergence is monitored by observing changes in the residuals of the conservation variables, the heat flux on the surface and variations in pressure values near the surface at the outflow plane. A local timestepping procedure that is based on the CFL and viscous stability requirements is used to accelerate convergence to steady-state and details of this procedure are found in reference 13. Typical computational speeds on the scalar code were about 2.E-04 CPUs/timestep/node and vectorization efforts are underway to enhance this speed.

An initial analysis of the complete flow domain resulted in inaccurate descriptions of the heating rates and surface pressures. This analysis indicated the need for the finite element mesh to have more elements at critical locations of the flow, such as, at the sharp leading edge and the corner to obtain satisfactory correlation with experimental data. The analysis described herein divides the flow domain into three regions. The three regions are shown in figure 5 and are categorized as : (a) the sharp leading edge section, (b) the flat plate section, and, (c) the ramp section.

\section{Sharp Leading Edge}

The finite element mesh that results after multiple refinements/derefinements depends a greal deal on the "skeleton" mesh used to start the adaptation procedure. A judicious selection of the skeleton mesh models the physics of the flow better and minimizes the number of elements used in the analysis. The skeleton mesh used to model the sharp leading edge is shown in Fig. 6 . The skeleton mesh was designed such that on refinement, aspect ratios of unity exist right at the leading edge and higher aspect ratios prevail downstream of the leading edge. The mesh obtained after three refinements on the initial mesh is shown in Fig. 7 (a) and contains 7245 nodes and 7235 elements (5781 quads and 1454 triangles). Density contours for the leading edge section of mesh in figure 7 (b) show good definition of the leading edge shock and smooth variation of density within the boundary layer. At the leading edge, the boundary layer displacement effect causes the density at the wall to increase by an order of magnitude and then to drop off quite rapidly away from the leading edge.

Details of the finite element mesh very near the sharp leading edge ( box $A$ in figure $7(a)$ ) is shown in figure $8(a)$ and the density contours on this section of the mesh appear in figure 8(b). It is interesting to note the "wiggles" that appear in the contours at $x=0.005$ where the element aspect ratio jumps by a factor of 20 in the flow direction. The effect of this jump is also seen in figure 9 in the distribution of the wall pressures near the leading edge.

A region of the mesh where the element aspect ratios change considerably normal to the flow direction is at $y=0.005$. This jump is inherited from the macroelements that constitute the skeleton mesh in figure 6 . The effect of the aspect ratio jump in the normal direction is best seen by focusing in on the region at the exit plane $(x=0.1)$ defined by box $B$ in figure $7(a)$. The finite element mesh at the outflow plane is shown in figure 10 (a) and the distribution of pressure along $y$ at $x=0.1$ is plotted in figure $10(\mathrm{~b})$. The presence of oscillations near the wall and at mesh transitions is very evident in this distribution. The bigger "wiggle" occurs where the element aspect ratio in the normal direction changes abruptly by a factor of ten. The analysis was repeated with both the second and fourth order dissipation turned off for elements located close to the wall and away from the vicinity of the sharp leading edge. This was possible using arrays that contain information about element neighbors. The distribution of pressure at the exit with no explicit dissipation close to the wall in shown in figure 10(c). The pressure jump at the wall is absent and pressure levels within the boundary layer are higher. However, the oscillations caused by the aspect ratio jumps are still present. The distribution of pressure at the exit, shown in figure 11, indicates the presence of pressure gradients normal to the wall even at regions close to the wall. Pressure, being derived from the conservation variables, appears to be more sensitive to mesh transitions than, say, density, the distribution of which is plotted in figure 12, exhibiting a smaller kink at $y=0.005$.

The surface pressure and heat transfer coefficient obtained from this analysis are compared to predictions by the interaction theories in figure 13. The results compare favorably, especially the heat transfer coefficient. Pressure levels close to the leading edge are well behaved and do not exhibit the "inverse spike" reported by other researchers 22 . The maximum deviation in pressure levels is seen to be near the leading edge.

It is instructive to take a quick look at the flow physics close to the leading edge. As seen in Figure 14, very close to the leading edge the flow is near free molecular flow and can be modelled onty on the basis of kinetic theory. The transitional layer has mixed kinetic and continuum properties. The merged layer which lies a little downstream of the transition layer can be described by the Navier-Stokes equations but needs velocity slip and temperature jump conditions at the wall23. Downstream of the merged layer, where a 
distinct inviscid layer develops between the shock and the viscous layer, is the interaction region. The NavierStokes equations with no-slip boundary conditions do not accurately model the physics of the flow up to this interaction region. So the accuracy of the finite element calculations very close to the leading edge is less than perfect and discrepancies on comparison with the strong interaction theories is not unexpected.

\section{Flat Piate Section}

Profiles of the conservation variables obtained at the exit of the leading edge section are used as inflow conditions to the flat plate section.

The skeleton mesh used to model the second section of the problem, the flat plate region, is shown in figure 15. After three overall refinements the elements at the wall were divided into 10 sub-elements according to equation (9). The grid parameter $\beta$ was set to be 1.02 and the subdivision yields elements with aspect ratios over 200 at the wall. The finite element mesh that results after three refinements on this initial mesh contains 8483 nodes and 8593 elements (5151 quads and 3442 triangles) and is shown in figure 16(a). Density contours on this mesh in figure 16(b) show the mesh capturing the leading edge shock and growth of the boundary layer along the plate. Details of the finite element mesh near the inflow (box $A$ in figure 16(a)) and the density contours on this section of the mesh appear in figures 17 (a) and 17(b). Density profiles at the inflow and the exit of the flat plate section, shown in figure 18, illustrate growth of the boundary layer and weakening of the leading edge shock as the flow traverses the plate.

A comparison of the density profiles at the outtlow of the leading edge and at inflow to the flat plate section in figure 19 indicates the adequacy of nodal distribution in the normal direction. Linear interpolation is used at the inflow of the flat plate section which tends to dampen some of the oscillations present at the exit of the leading edge section as illustrated by the pressure profiles in figure 20 . It is to be noted that these oscillations become fixed at the inflow planes, and hence will influence subsequent analyses.

As with the leading edge section, profiles on the exit plane $(x=0.7)$ of the flat plate section are used to define the inflow boundary of the ramp section.

\section{Ramp Section}

The skeleton mesh used for modelling the ramp section of the flow domain is shown in figure 21. After three overall refinements on this skeleton mesh a layer of structured quadrilateral elements were generated at the wall. Each element at the wall was divided into ten sub-elements with a stretching factor of 1.03. The mesh that results after three refinements on this initial mesh is shown in figure 22(a). Density contours obtained on this mesh appear in figure 22 (b) and salient characteristics of the flow such as the leading edge, induced and resultant shocks are all seen to be well captured. The ability of the adaptive refinement procedure to locate regions of high gradients is exhibited by taking a closer look at the mesh in the ramp comer (box $A$ in figure 22(a)). The mesh in figure 23(a) and density contours on this mesh in figure $23(\mathrm{~b})$ illustrate capture of the leading edge shock and compression of flow that results in the induced shock downstream of this region. The fine mesh at the surface is needed to model separation and subsequent reattachment of the boundary layer in the vicinity of the corner. Explicit dissipation terms for elements that are located close to the surface were zeroed out to ensure accurate computation of surface quantities.

Details of the flowfield, especially the coupling between the inviscid and viscous regions of flow, are best described by profiles of select variables at specified axial locations. Figure 24 indicates the various $x$-stations wherein the variation in $y$ direction of quantities such as pressure and temperature are studied. Locations indicated by $A, B, C$ and $D$ are used to describe flow details in the domain up to the comer while locations $E$ to $\mathrm{H}$ detail flow characteristics up the ramp.

The distribution of pressure normal to the surface at locations $A$ to $D$ is shown in figure 25. As the flow moves from the inlet toward the corner, the presence of the adverse pressure gradient due to the ramp is clearty seen. Fluid in the vicinity of the corner cannot overcome the effects of the pressure gradient as well as the skin friction at the wall and flow separation results. The separated boundary layer becomes a free shear layer outside of the recirculation region. Pressure profiles in figure 25 and velocity profiles in figure 26 illustrate this effect. The boundary layer separation is clearly seen by reversal in flow direction of the u-velocity profiles. The distribution of temperature at various $x$-stations in figure 27 shows evidence of increase in boundary layer thickness as the flow approaches the ramp corner. Gradients of the temperature profiles at the surface indicate the decrease in heat transfer rates from location A to location D.

Characteristics of the flow up the ramp are described by the distributions at locations $E$ to $H$. Pressure profiles at E, F, and $G$ in figure 28 indicate the strengthening of the induced shock and the change in location of the leading edge shock relative to the surface. The distribution of pressure at exit of the ramp (location $\mathrm{H}$ ) shows the resultant shock which is derived from the interaction of the leading edge shock with the induced shock. A weak expansion fan also results from this interaction, the effect of which is seen by the small drop in surface pressures at location $\mathrm{H}$ relative to that at $\mathrm{G}$. The distribution of density in figure 29 indicates the thinning of the boundary layer as the flow moves up the ramp. This reduction in thickness of the boundary layer is also seen in figure $\mathbf{3 0}$ which shows $U$-velocity profiles at various $x$-stations. The presence of the recirculation is seen by the flow reversal close to the surface at station $E$. At the exit from the ramp section, the boundary layer profile is fully developed and downstream of this region the interaction between the inviscid and viscous regions of the flowfield is negligible. Temperature profiles at locations up the ramp, shown in figure 31, reflect the "thinning" of the boundary layer. The largest gradient in temperature at the surface is seen to be at location $G$ indicating the approximate location of peak heating rates. 
Heat flux, skin friction and pressure coefficients for the ramp surface obtained from the finite element approach are compared to experimental observations of Holden21. As mentioned earlier, flow conditions used in the finite element analysis were conditions representative of runs 19 and 21 in the Calspan tunnel. Figure 32 compares the finite element predictions for heat transfer coefficient to those obtained from tunnel runs. Heat transfer coefficients computed from the finite element scheme compare well with both the experimental runs for locations upto the corner as well as halfway up the ramp. Heating rates measured near the exit of the ramp for run 19 are about twice that of both run 21 and numerical predictions. The finite element predictions seem to compare better with experimental data of run 21 as seen by figure 33 . The heat transfer coefficients match quite well at the separation and reattachment regions and the values of the peak heating rate are in excellent agreement. The main discrepancies seem to be location of the peak heat rate, which for the analysis is shifted to the left, and a steeper drop in heating rates downstream of this location.

Skin friction coefficients calculated from the finite element approach are compared to those measured in tunnel runs 19 and 21 in figure 34. Levels of the skin friction coefficient compare well with the experimental data for regions unaffected by the recirculation while the size of the recirculation region is underpredicted by the finite element approach. Both experimental runs measure flow separation at around $x=15^{\prime \prime}$ while the finite element predictions locate separation at $x=16^{*}$. The location of the reattachment point from run 21 is $x=20.3^{*}$ and the finite element procedure locates this point at $x=19.3^{n}$. Unsteadiness of the recirculation bubble, which could account for the discrepancies in the finite element predictions, was not considered in this analysis.

A comparison of surface pressure coefficients using the finite element approach with data from experimental runs 19 and 21 appears in figure 35 . Pressure levels predicted by the analysis is seen to be lower at the inflow and recirculation regions. The rise in pressure up the ramp is much more rapid with the final surface pressure falling midway between pressure levels of runs 19 and 21 . The experimental data shows sharp peaks in pressure coefficients near the exit while the finite element approach indicates a gradual downward trend. This implies that the interaction between the leading edge shock and induced shock is stronger than that predicted by the finite element approach. The pressure rise for Mach 11.7 inviscid flow over a $15^{\circ}$ ramp is about 17.5 and the finite element analysis yields a peak pressure rise of over 22 .

\section{Rofinement Indicators Revisited}

The finite element meshes for analyzing flow over the ramp were obtained by multiple refinements on the initial meshes, using the inviscid indicator, with pressure as the key variable, and boundary layer indicator. Proper selections of the key variable and values of $\alpha$ and $\beta$, the refinement and derefinement thresholds, impact heavily on the quality of the resulting finite element mesh. The effect of refinement and threshold parameters on the finite element mesh for the ramp section merits investigation. Meshes that result from refinements are evaluated by comparing nodal distribution in the $y$-direction for some specified $x$ station.

Four cases of interest, obtained by varying the adaptation parameters are described below.

Case 1: Key variable - pressure, $\alpha=15, \beta=1$, viscous refinement indicator off.

Case 2: Key variable - pressure, $\alpha=15, \beta=.1$, viscous $\alpha=.15$.

Case 3: Key variable - density, $\alpha=.15, \beta=.1$, viscous refinement indicator off.

Case 4: Key variable - density, $\alpha, \beta=1$, viscous refinement indicator off.

The pressure profile at location A (figure 24) in the flat portion of the ramp for the initial mesh is seen in figure 36. For both case 1 and case 2, the use of pressure as the key variable causes the leading edge shock to be refined. The profile reflecting case 2 also shows nodes being added inside the boundary layer. The location of these new nodes are seen in figure 37 from the u-velocity profiles. The viscous indicator is seen to add elements where the velocity profile is essentially linear.

The use of density as the key variable (case 3) indicated by the profile in figure 36 shows refinements at the shock and the boundary layer. Elements within the boundary layer are refined due to sharp changes in the density profile as seen in figure 38. The effect of lowering the refinement threshold is shown in figure 39 where the pressure profile that results from case 4 is compared to that of case 3. More elements at the leading edge shock are refined for case 4 and the pressure profile is very similar to that obtained using pressure as the key variable and the viscous indicator (case 2) in figure 36.

The profiles in figures $36-39$ illustrate the inadequacy of using equation (4) with pressure as the key variable to capture details of the boundary layer. The use of density as the key variable with appropriate threshold values causes refinements at the shock as well as within the boundary layer. The use of the viscous indicator equation (8) leads to excessive refinements within the boundary layer, where the velocity profile is essentially linear.

Oscillations in pressure close to the wall is observed in figures 36 and 39 . The pressure levels of the added nodes are higher than the values at the adjacent nodes. The inaccuracy is due to pressure being derived from conservation variables while conservation variables at a new node are obtained by averaging variables at adjacent nodes. A time history of pressure at such a typical node in figure 40 shows the nodal pressure dropping down to the right levels within 
200 iterations on this mesh. A procedure based on interpolating primitive variables at each refinement level was also tried. This scheme eliminates inaccurate pressures at new nodes within the boundary layer but shows minimal improvements on convergence rates. Interpolation techniques are being investigated to eliminate this inaccuracy and help increase convergence rates.

\section{CONCLUDING REMARKS}

An adaptive finite element procedure that uses both triangles and quadrilateral elements is coupled with a multi-step Galerkin-Runge Kutta algorithm to model laminar hypersonic compressible flows. The finite element mesh is adapted in regions of high gradients by the use of inviscid and viscous refinement indicators. The facility to add in layers of structured quadrilateral elements at the wall for better representation of the boundary layer is included within the tramework of the program.

The ability of the adaptive finite element procedure to capture details of compressible flow with strong-inviscid viscous interactions is demonstrated by modelling the Mach 11.7 flow over a ramp. To ensure adequate resolution of complex flow features, the flow domain is split up into three sub-regions: (a) the sharp leading edge, (b) the flat plate region, and (c) the ramp section.

The accuracy of the finite element solution at the sharp leading edge section is evaluated by comparison with predictions of strong interaction theories. The complete theory of Bertram and Blackstock yield estimates for surface pressure distributions while the heat-transfer coefficient is obtained from the strong interaction theory of $\mathrm{Li}$ and Nagamatsu. Good correlations between the finite element predictions and the strong interaction theories are obtained for the leading edge section.

The finite element mesh for the ramp section captures the physics of the complicated viscousinviscid interactions at the corner. Surface coefficients of heat transfer, skin friction and pressure obtained from the finite element calculations compare well with the experimental measurements of Holden. The effect of the leading edge shock on the surface is seen by the peak in heating rates up near the exit section of the ramp.

Smooth mesh transitions within the boundary layer is seen to be important to model the flow behavior without any localized "wiggles" in the solution. Elimination of all explicit dissipation within the boundary layer is essential to obtain accurate predictions of heat transfer, skin friction and pressure coefficients. The refinement indicator used to key in on the boundary layer needs to be modified to ignore enrichment at regions where the velocity profiles are linear. The ability to pick out elements that lie in locations of flow reversals is also needed for flow domains that include separation and reattachment regions. A refinement procedure that puts in an excessive number of elements at the surface is to be avoided since this leads to increased computational effort and slower convergence rates.

The study highlights some of the complexities involved in modelling compressible viscous flow problems, especially, high speed flows with strong shock-boundary layer interactions. The physics of the flow as well as surface coefficients obtained from the adaptive finite element approach compare favorably with theoretical and experimental data. This trend is encouraging and the procedure indicates good potential for accurate aerothermal load predictions in the design of high speed vehicles.

\section{REFERENCES}

${ }^{1}$ Lohner, R., Morgan K., and Zienkiewicz, O. C.: "The Solution of Non-Linear Hyperbolic Equation Systems by the Finite Element Method," Int. J. Num. Meth. Fluids Vol. 4 (1984), pp. 1043-1063.

2Bey, K. S., Thornton, E. A., Dechaumphai, P., and Ramakrishnan, R.: "A New Finite Element Approach for Prediction of Aerothermal Loads-Progress in Inviscid Flow Computations," presented at the AIAA 7th Computational Fluids Dynamics Conference, Cincinnati, Ohio, July 15-17, 1985, AIAA Paper No. 85-1533.

3Thornton, E. A., Ramakrishnan, R., and Dechaumphai, P.: "A Finite Element Approach for Solution of the 3D Euler Equations," presented at AIAA 24th Aerospace Sciences Meeting, Reno, Nevada, January 6-8, 1986, AlAA-86-0106.

4 Dechaumphai, P., Thornton, E.A. and Wieting, A.R.:"Flow-Thermal-Structural Study of Aerodynamically Heated Leading Edges," Presented at the AIAA/ASME/ASCE/AHS 29th Structures, Structural Dynamics and Materials Conference, Williamsburg, Virginia, April 18-20, 1988.

5Zienkiewicz, O. C., Lohner, R., and Morgan, K.: "High Speed Inviscid Compressible Flow by the Finite Element Method," presented at the MAFELAP-V Conference, May 1-4, Brunel, UK.

6stewart, J.R., Thareja, R.R., Wieting, A.R. and Morgan, K.: "Application of Finite Element and Remeshing Technique to Shock Interference on a Cylindrical Leading Edge", Presented at the AIAA 26th Aerospace Sciences Meeting, Jan 11.14, 1988, Reno, Nevada. AIAA-88-0368.

7Lohner, R.: "The Efficient Simulation of Strongly Unsteady Flows by the Finite Element Method," Presented at the AlAA 25th Aerospaces Sciences Meeting, Reno, Nevada, January 12-15, 1987, AlAA -870555.

8Oden, J. T., Demkowicz L., Strouboulis, T., and Devloo, P. : "Adaptive Methods for Problems in Solid and Fluid Mechanics," in I. Babuska , O. C. Zienkiewicz, J. P.de S. R. Gago, and A. de Oliveira , (Eds.), 
Adaotive Methods and Error Refinement in Finite Element Computation, John Wiley and Sons, Ltd., London 1986.

9Shapiro, R. A., and Murman, E. M.: "Cartesian Grid Finite Element Solutions to the Euler Equations," presented at the AIAA 25th Aerospace Sciences Meeting, Reno, Nevada, January 12-15, 1987, AIAA-87. 0559.

10Peraire, J. Vahdati, M. Morgan, K. , and Zienkiewicz O. C. : "Adaptive Remeshing for Compressible Flow Computations," Journal of Computational Physics, 72, 449-466, 1987.

11Thareja, R.R., Stewart, J.R., Hassan, O., Morgan, K., and Peraire, J.: "A Point Implicit Unstructured Grid Solver for the Euler and Navier-Stokes Equations," Presented at the AIAA 26th Aerospace Sciences Meeting, Jan 11-14, 1988, Reno, Nevada. AIAA-880036.

12Ramakrishnan, R., Bey, K.S., and Thornton, E.A.: "An Adaptive Quadrilateral and Triangular Finite Element Scheme for Compressible Flows," Presented at the AIAA 26th Aerospace Sciences Meeting, Jan 11-14, 1988, Reno, Nevada. AIAA-88-0033.

13Thomton, E.A., Dechaumphai, P., and Vemaganti, G.: "A Finite Element Approach for Prediction of Aerothermal Loads," Presented at the AIAANASME 4th Fluid Mechanics, Plasma Dynamics and Lasers Conference, May 12-14, 1986, Atlanta, GA. AIAA-861050.

14Roberts, G.O.: "Computational Methods for Boundary Layer Problems," Proceedings of the Second International Conference in Numerical Methods in Fluid Dynamics, Lecture Notes in Physics, Vol. 8. SpringerVerlag, NY, pp 171-177.

$15 \mathrm{Li}$, T.Y. and Nagamatsu, H.T., Journal of Aeronautical Sciences, Vol. 20, No. 5, 1953, pp. 345355.

16Lees, L. and Probstein, R.F.: "Hypersonic Viscous Flow over a Flat Plate", Report No. 195 (Contract AF 33(038)-250), Aero. Eng. Lab., Princeton Univ., April, 1952.

17Hayes, W.D. and Probstein, R.F.: "Hyoersonic Elow Theory", pp. 341-353, Academic Press, Inc., Now York, 1959.

18 Bertram, M.H. and Blackstock, T.A.: "Some Simple Solutions to the Problem of Predicting Boundary-Layer Self-Induced Pressure, NASA TN D798, April 1961.

19Dorrance, W.H.: "Viscous Hypersonic Flow," pp 154-157, McGraw-Hill Book Company, 1962.
20Holden, M.S.: "A Study of Flow Separation in Regions of Shock Wave-Boundary Layer Interaction in Hypersonic Flow," Presented at the AIAA 11th Fluid and Plasma Dynamics Conference, Seattle, Wash., July 1012, 1978.

21Calspan Corporation, "Laminar Flow Data Base," August 26, 1987.

22 Ray, R., Erdos, J. and Pulsonetti, M.V.: "Hypersonic Laminar Strong Interaction Theory and Experiment Revisited Using a Navier-Stokes Code," Presented at the 19th Fluid Dynamics, Plasma Dynamics and Lasers Conference, June 8-10, 1987. Honolulu, Hawaii.

23Tannehill, J.C., Mohling, R.A., and Rakich, J.V.: "Numerical Computation of the Hypersonic Rarefied Flow Near the Sharp Leading Edge of a Flat Plate," Presented at the AIAA 11th Aerospaces Sciences Mooting, Washington, D.C., January 10-12, 1973.

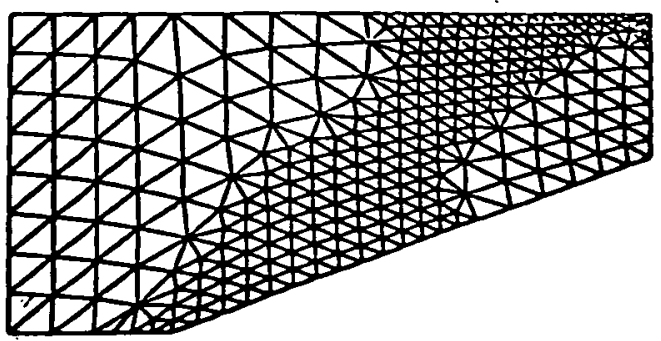

(a) Triangular enrichment

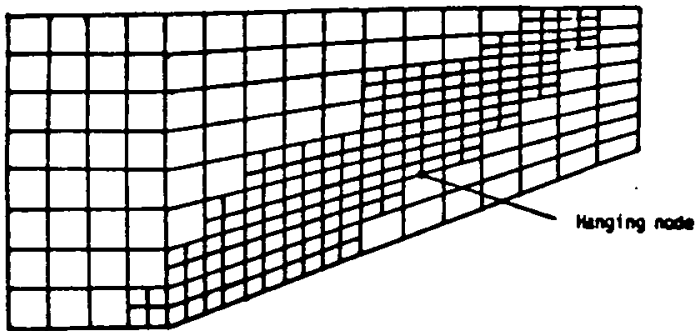

(b) Quad enrichment

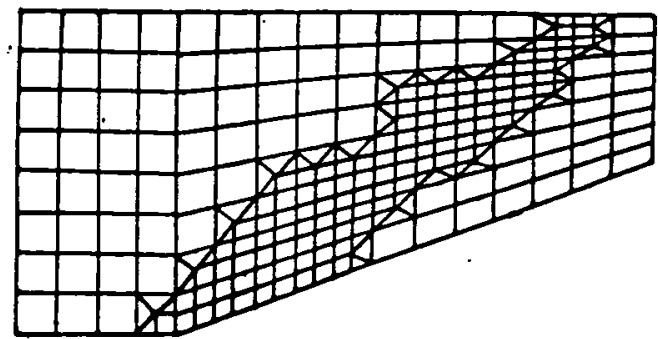

(c) Quad enrichment with triangular transition

Fig. 1 Mesh Enrichment using quadrilateral and triangular elements. 


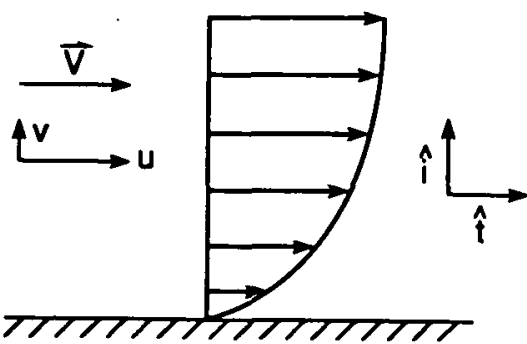

Fig. 2 Velocity profile at wall and unit vector defining change of velocity.

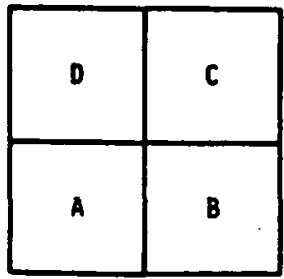

(a) Original Mesh

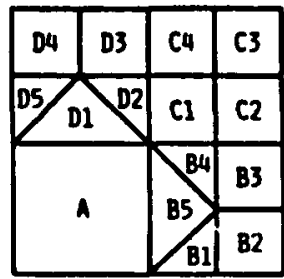

(b) Refinement of elements B, C, and D

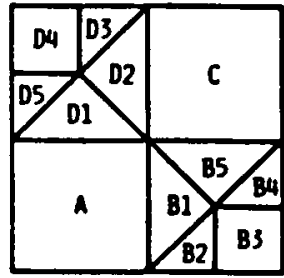

(c) Derefinement of element C

Fig. 3 Example of adaptation procedure using triangular transition elements.

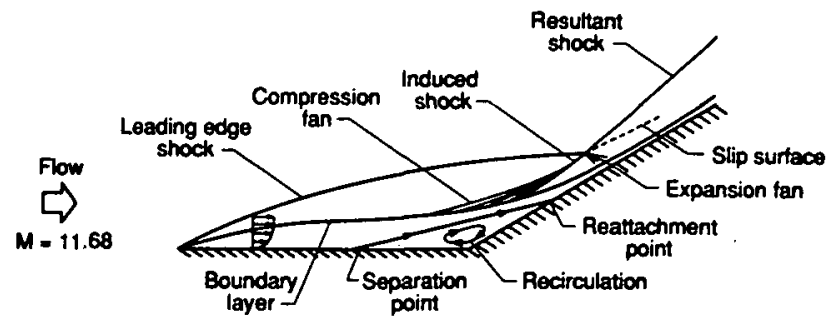

Fig. 4 Hypersonic flow over a $15^{\circ}$ ramp.

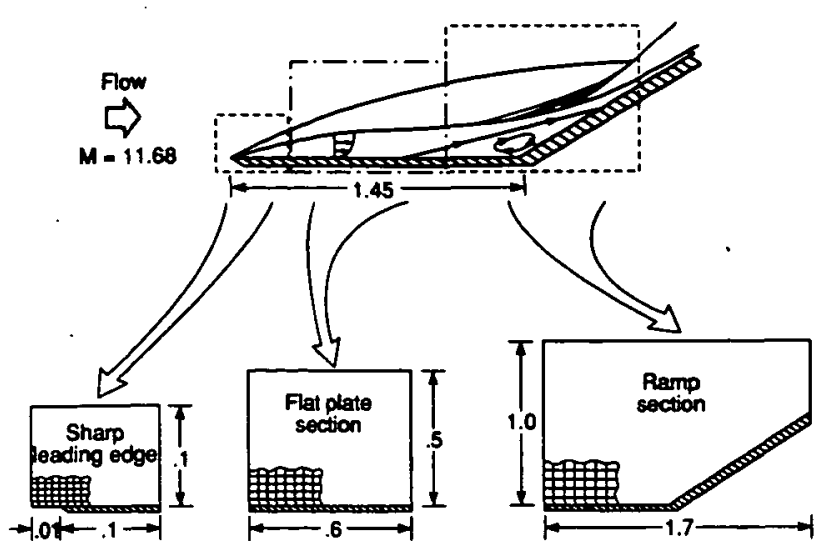

Fig. 5 Subdivision of hypersonic flow domain.

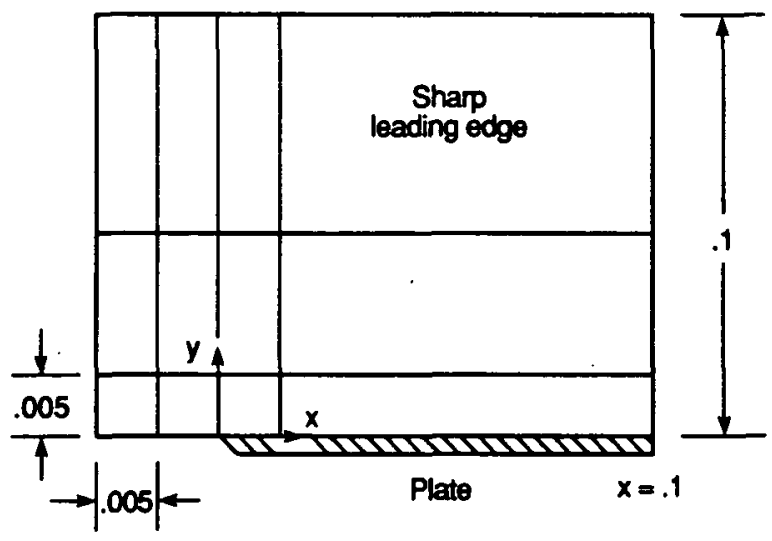

Fig. 6 Skeleton mesh for sharp leading edge.

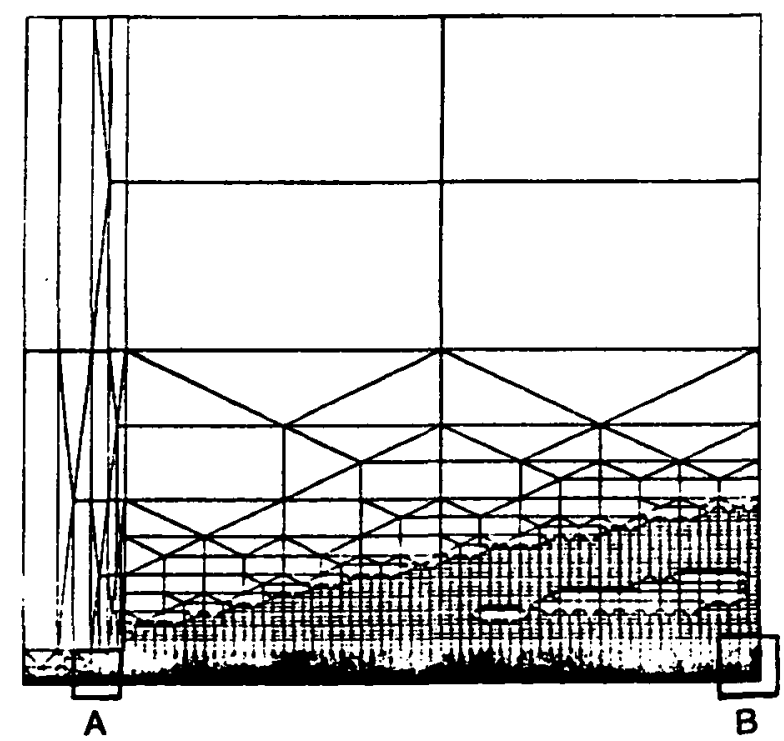

(a) Finite element mesh

Fig. 7 Finite element mesh and density contours for sharp leading edge. 


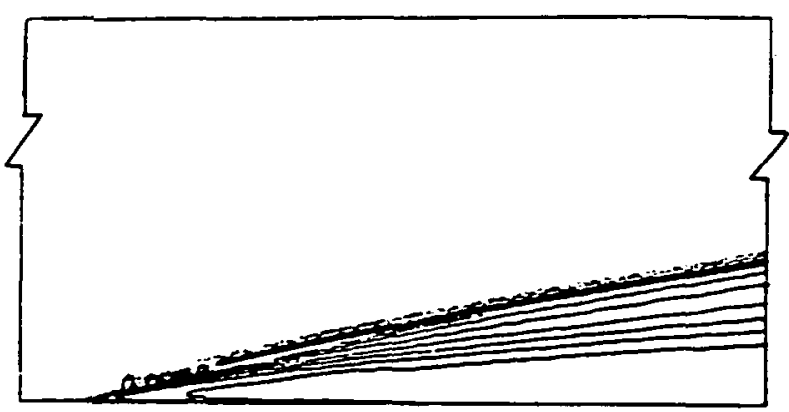

(b) Density contours

Fig. 7 Finite element mesh and density contours for sharp leading odge.

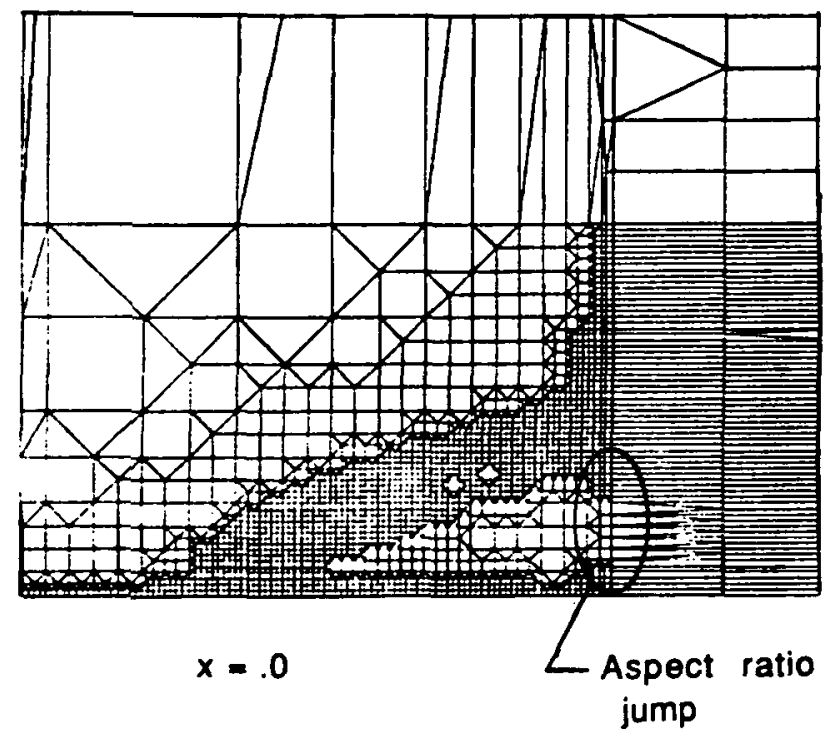

(a) Finite element mesh

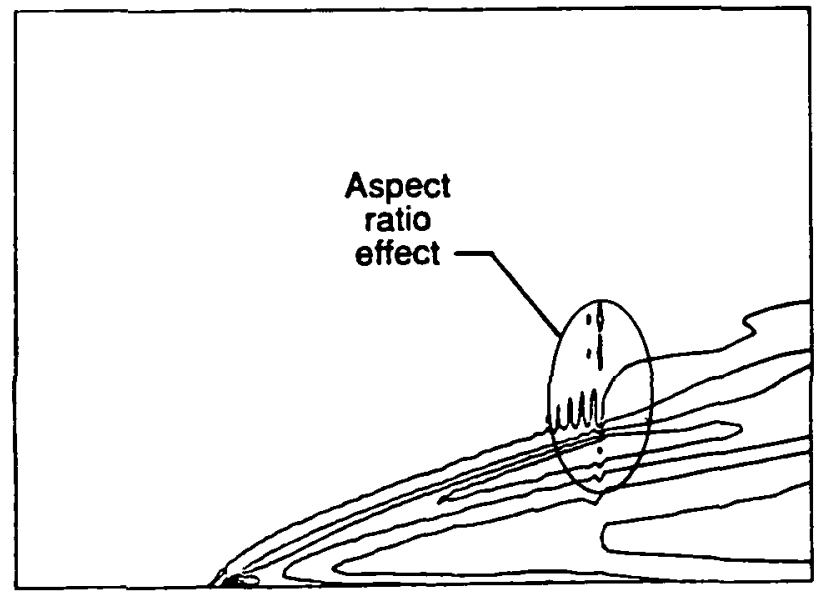

(b) Density contours

Fig. 8 Finite element mesh in the vicinity of the leading edge.

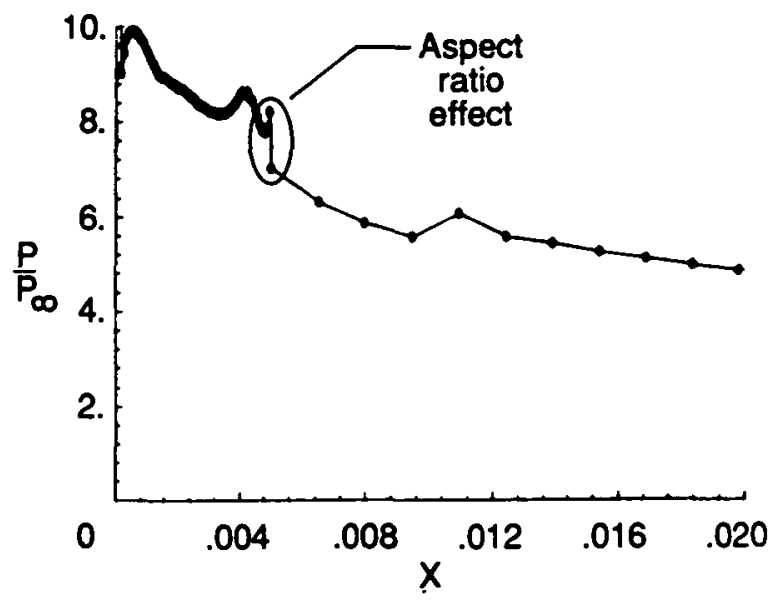

Fig. 9 Pressure distribution in the vicinity of the leading edge.

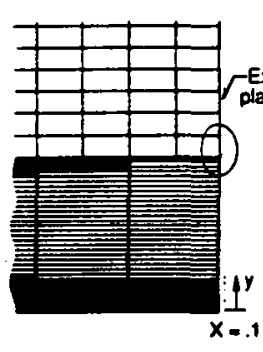

(a)

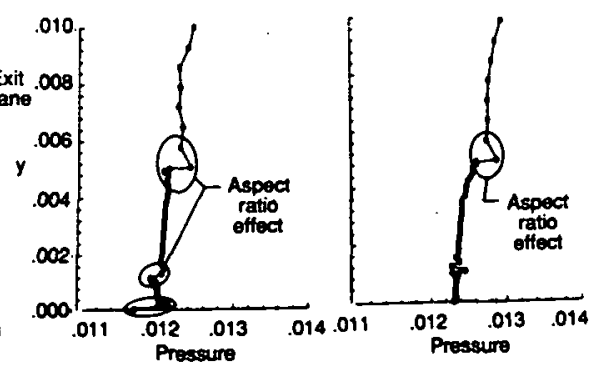

(b)

(c)
Fig. 10 Finite element mesh and pressure distribution at the exit plane of sharp leading odge.

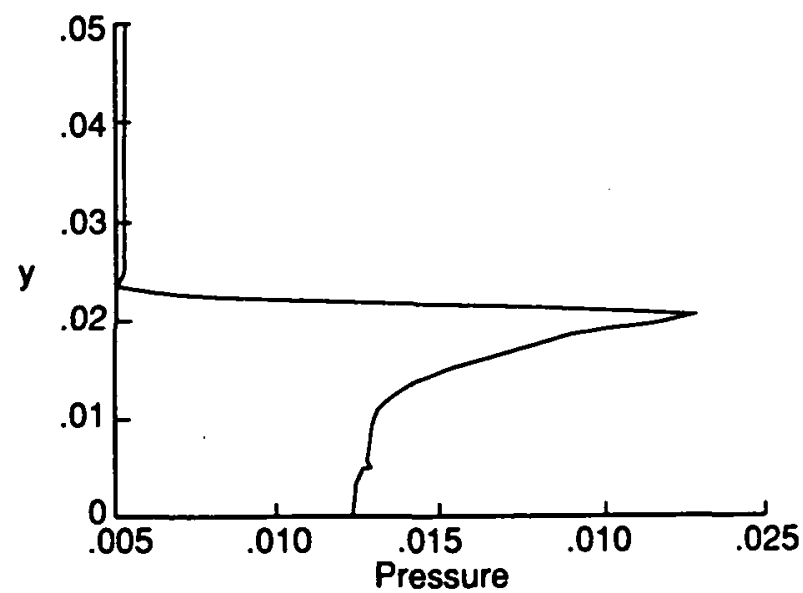

Fig. 11 Pressure distribution at exit plane of the leading edge section. 


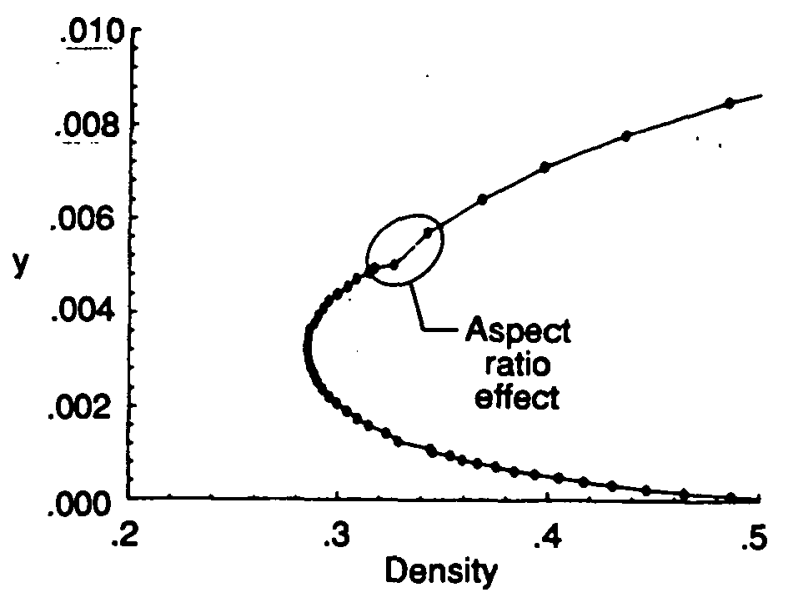

Fig. 12 Density distribution at ext plane of the leading edge soction.

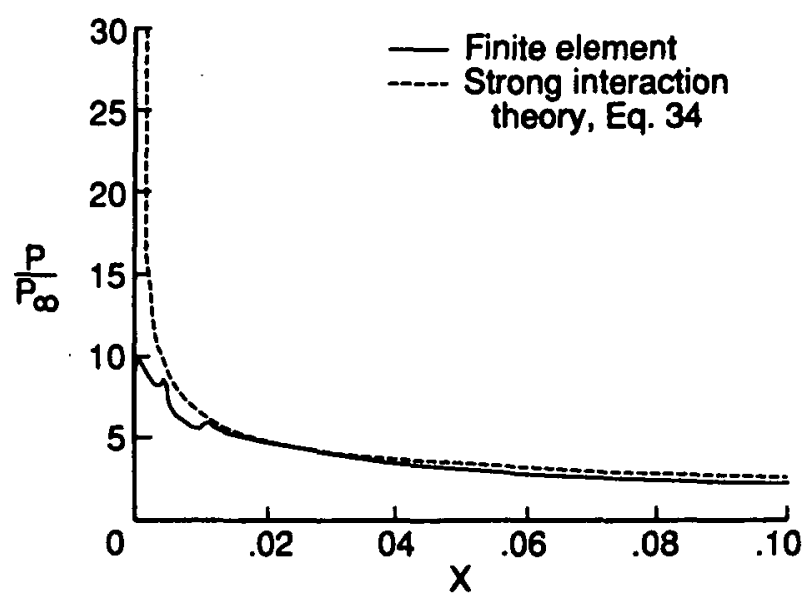

(a) Surface pressure ratio

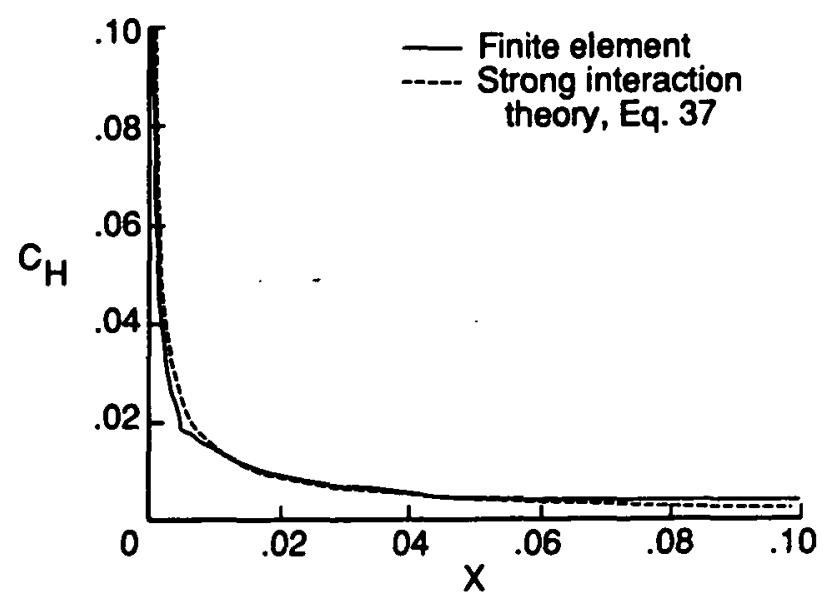

(b) Heat transfer coefficient

Fig. 13 Comparison of pressure and heat transter coefficients for the sharp leading edge.

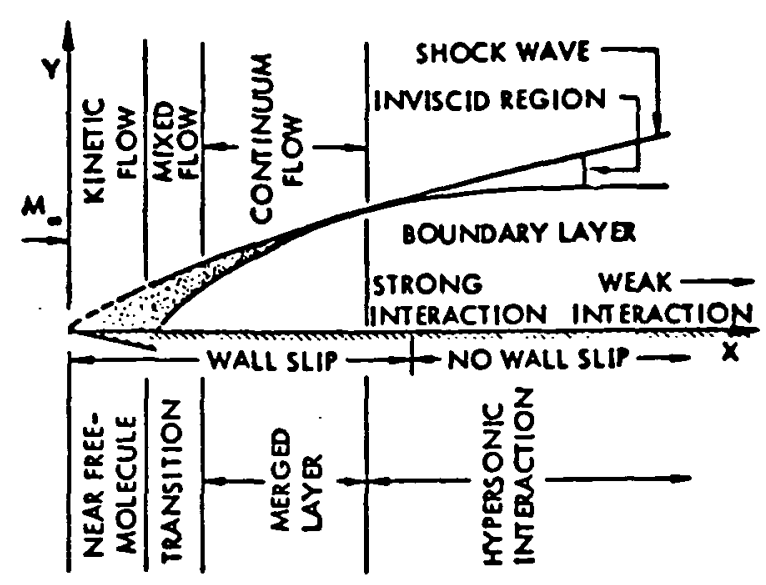

Fig. 14 Hypersonic flow detail at sharp leading odge.

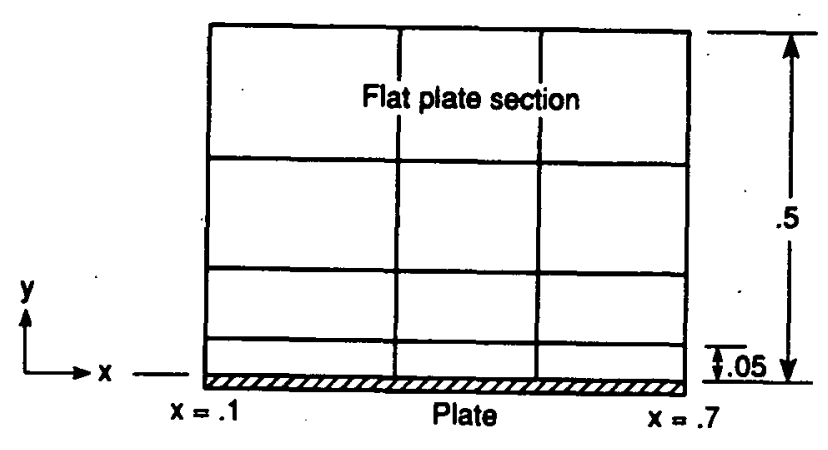

Fig. 15 Skeleton mesh for flat plate section.

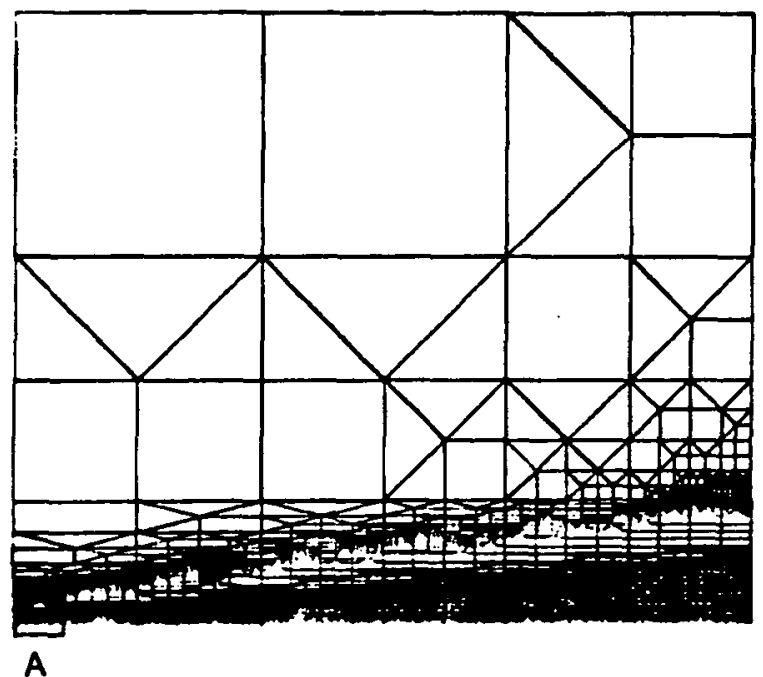

(a) Finite element mesh

Fig. 16 Finite element mesh and density contours for flat plate section. 


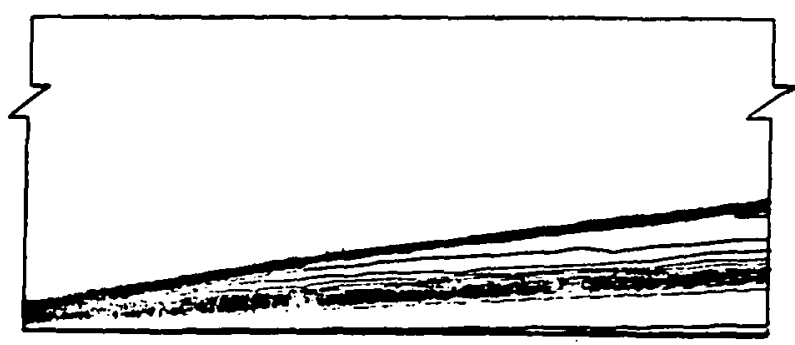

(b) Density contours

Fig. 16 Finite element mesh and density contours for flat plate section.

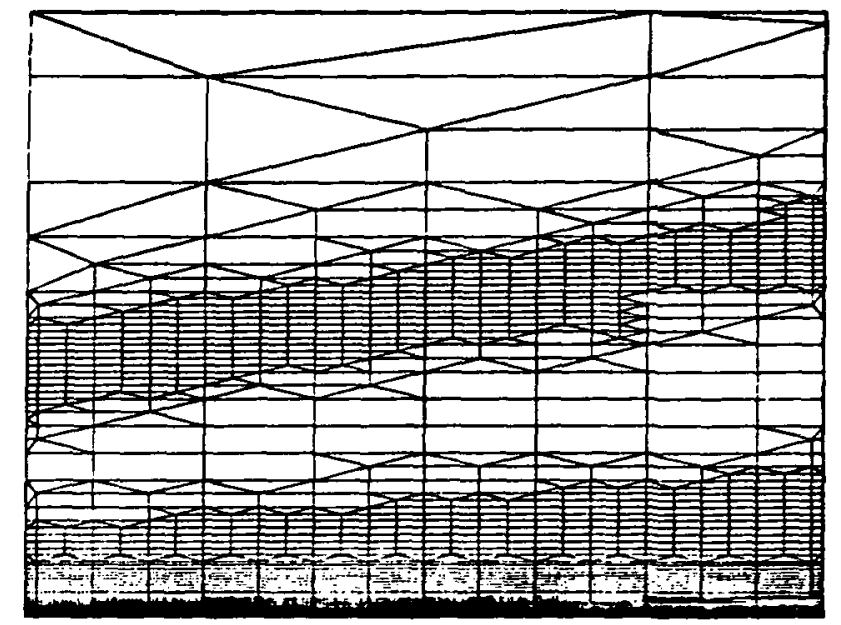

$x=.11$

$$
x=.15
$$

(a) Finite element mesh at inflow

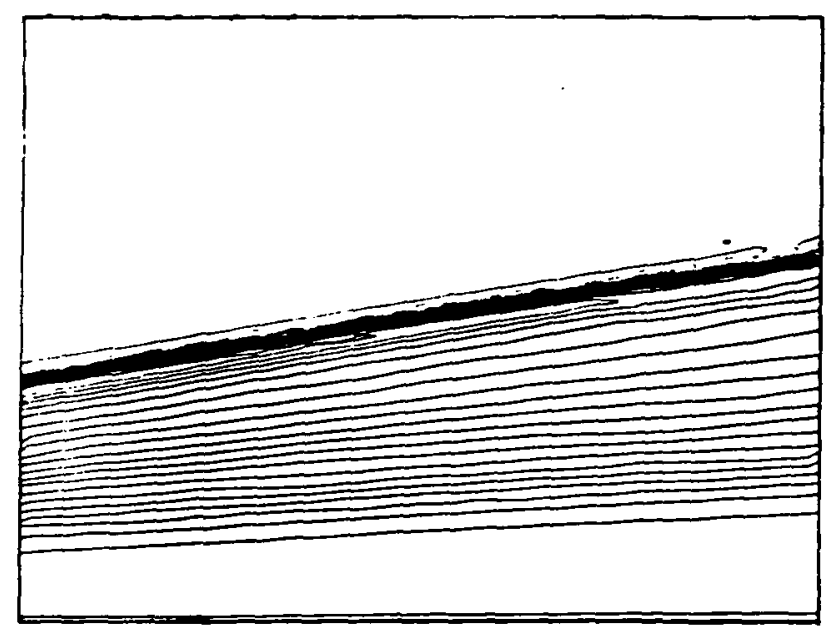

(b) Density contours at inflow section

Fig. 17 Detailed mesh at inflow for flat plate section.

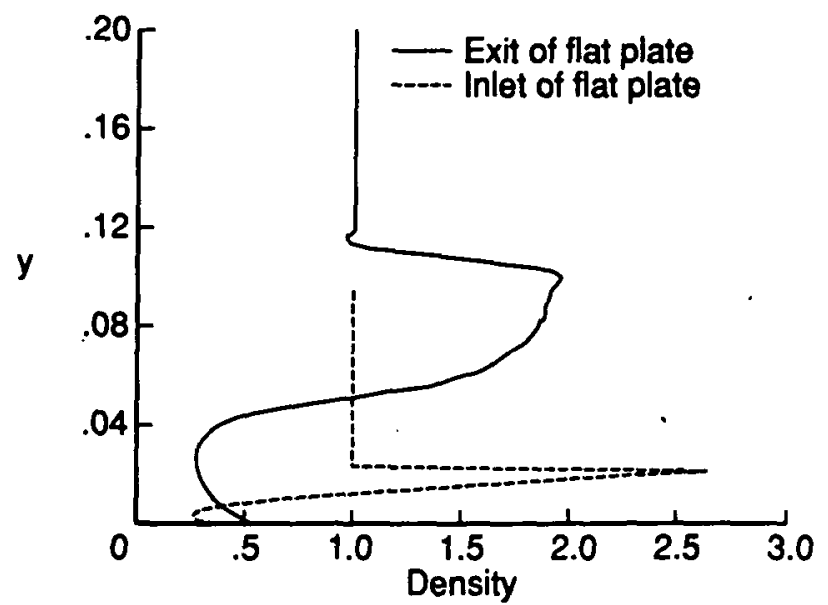

Fig. 18 Comparison of inlet and exit profiles of flat plate section.

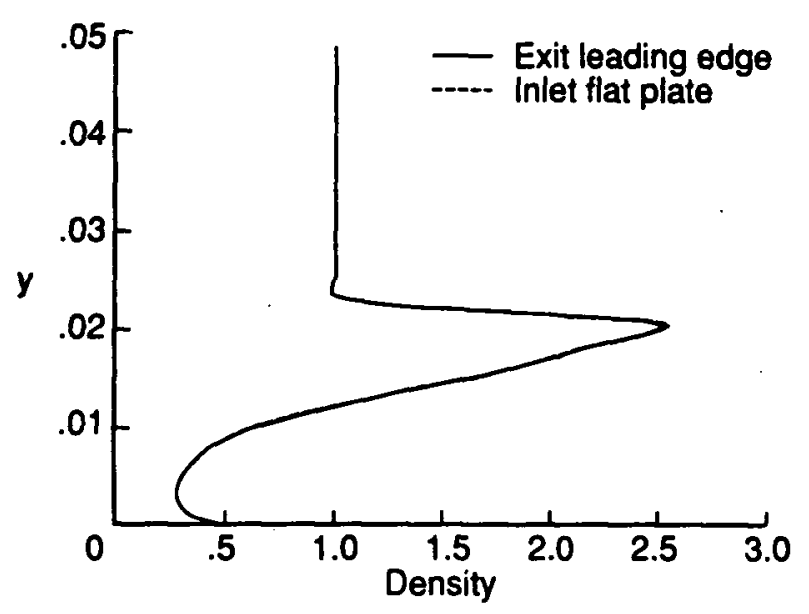

Fig. 19 Density profiles at exit of the leading edge and inflow to the flat plate section.

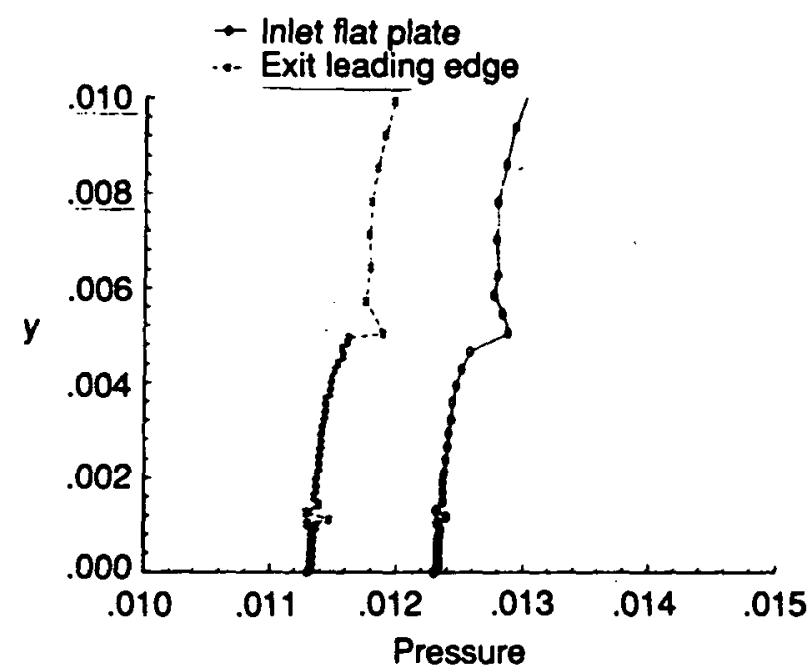

Fig. 20 Effect of interpolation procedure on pressure profiles at inflow to flat plate soction. 


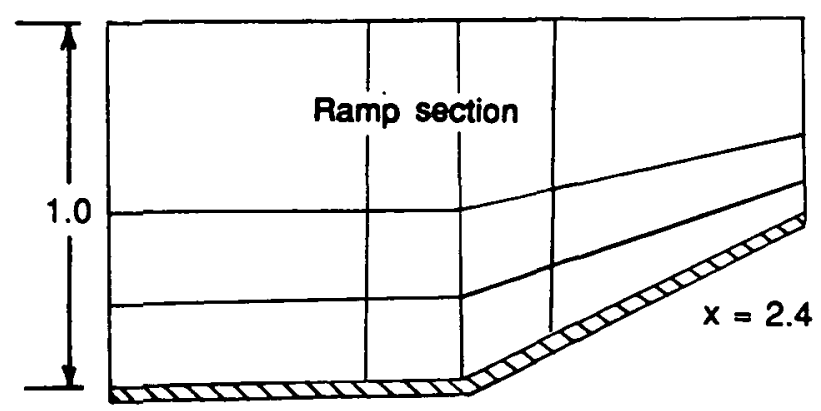

$x=0.7$

$x=1.45$

Fig. 21 Skeleton mesh for ramp section.

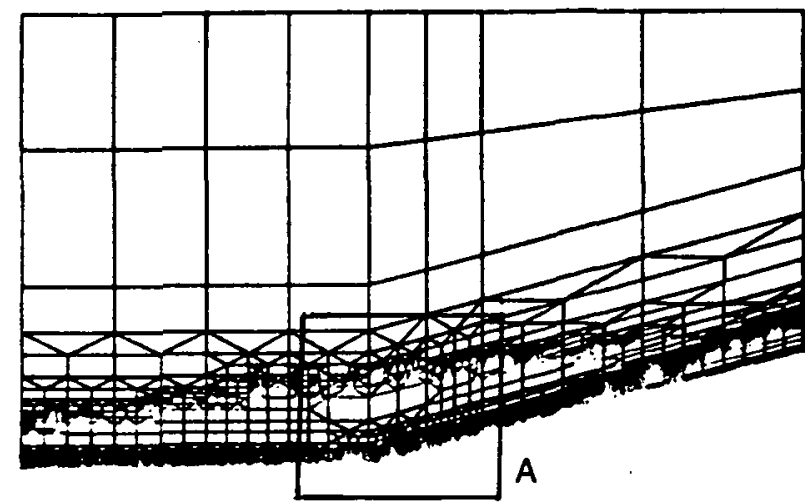

(a) Finite element mesh

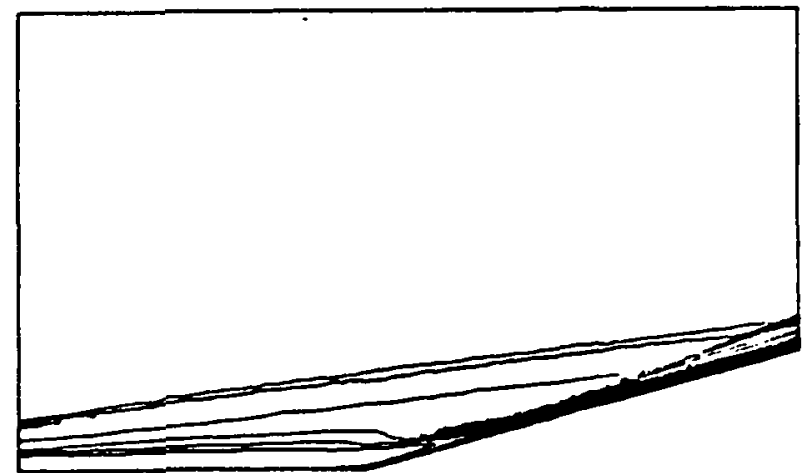

(b) Density contours

Fig. 22 Finite element mesh and density contours for ramp section

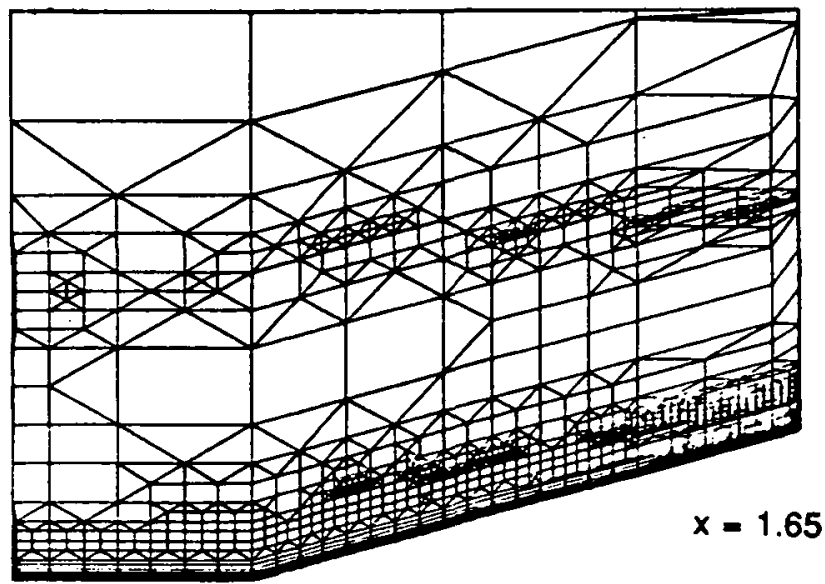

$x=1.3$

(a) Finite element mesh

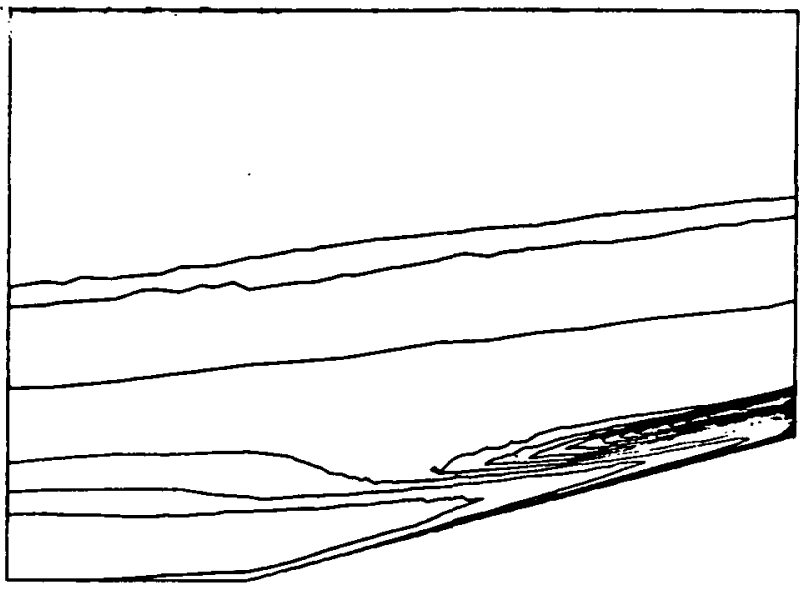

(b) Density contours

Fig. 23 Mesh detail at comer of ramp.

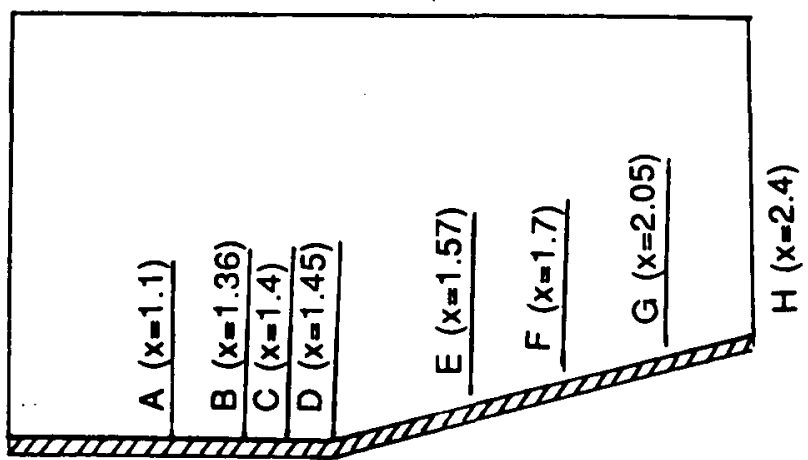

Fig. 24 Locations of profile stations along the ramp. 


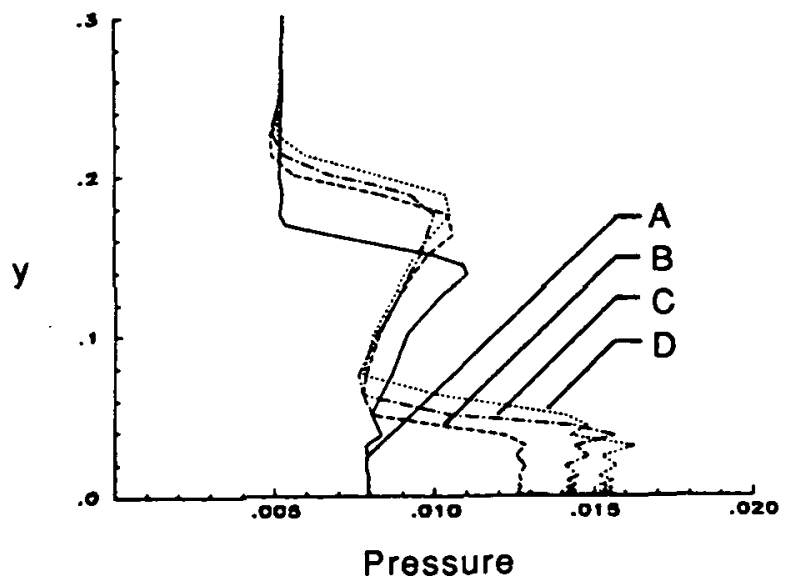

Fig. 25 Pressure distribution at locations on flat portion of ramp.

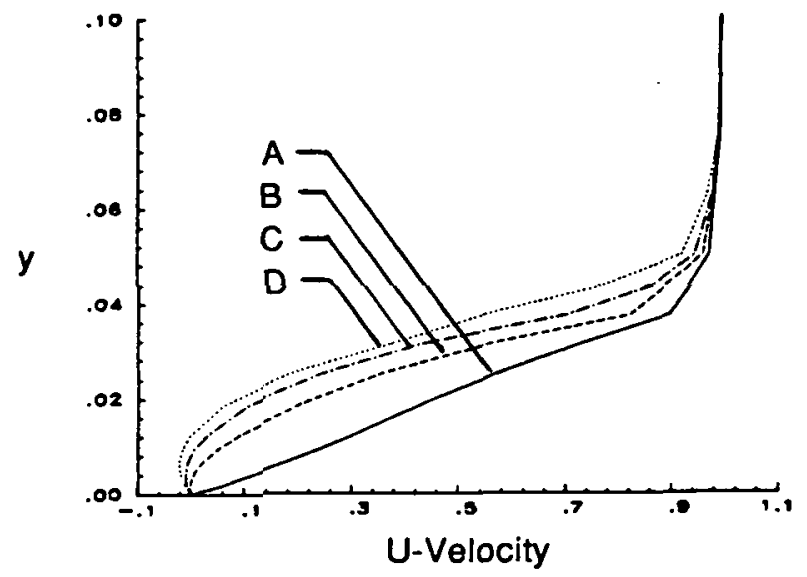

Fig. 26 Velocity profiles at locations on flat portion of ramp.

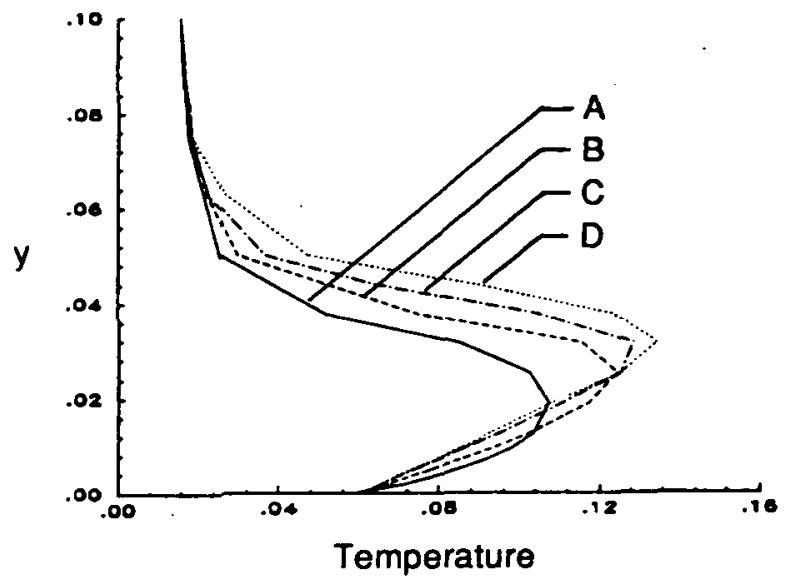

Fig. 27 Temperature distribution at locations on flat portion of ramp.

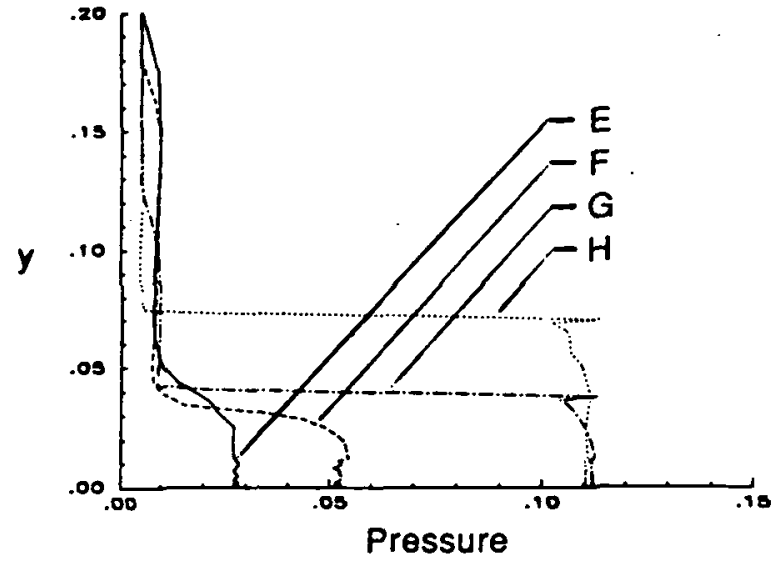

Fig. 28 Pressure profiles at locations up the ramp.

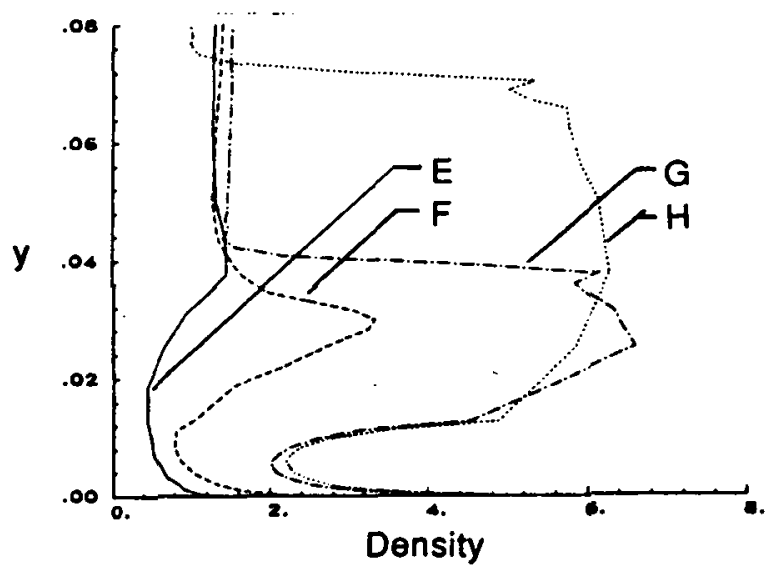

Fig. 29 Density profiles at locations up the ramp.

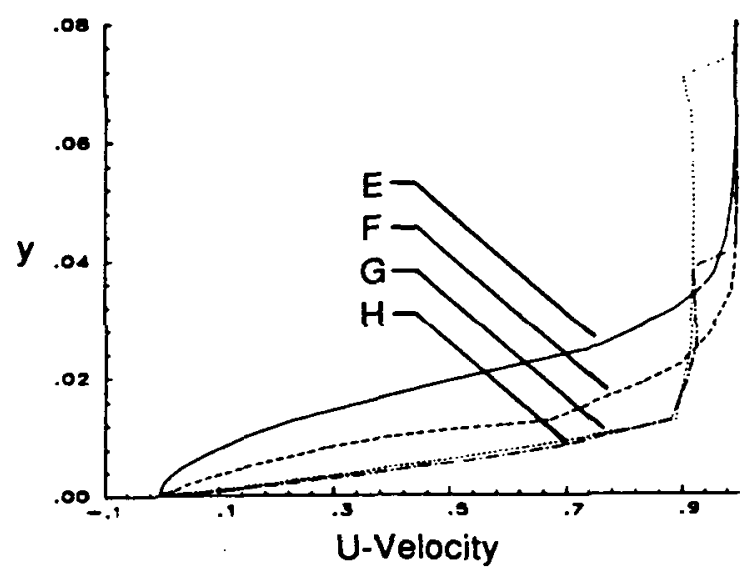

Fig. 30 Velocity profiles at locations up the ramp. 


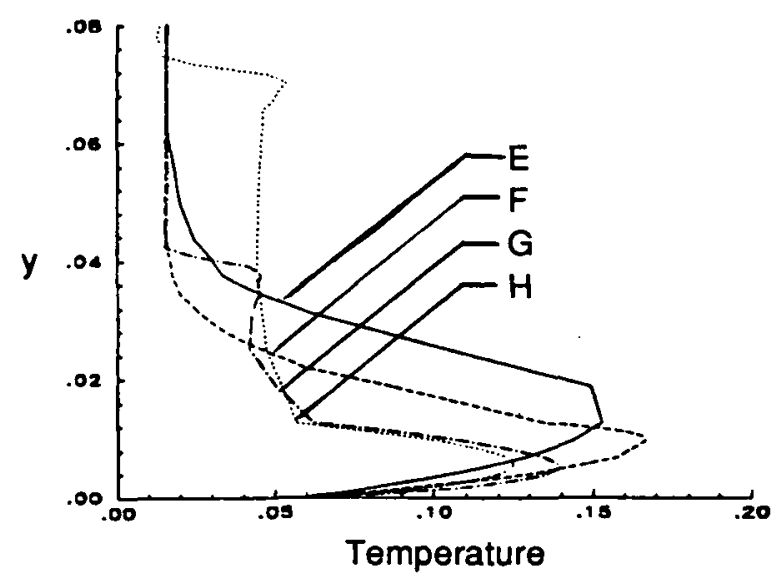

Fig. 31 Temperature profiles at loactions up the ramp.

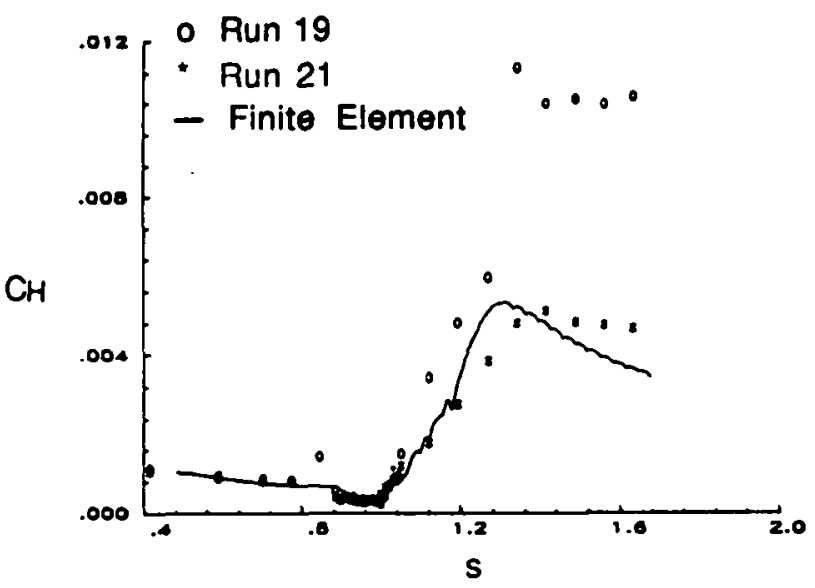

Fig. 32 Comparison of heat transfer coefficients on the ramp surface.

- Run 21

- Finite Element

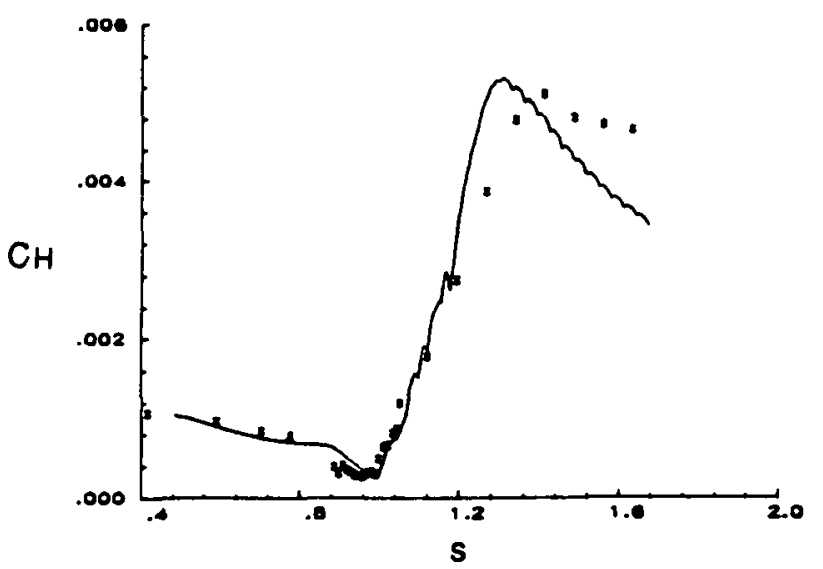

Fig. 33 Comparison of heat transfer coefficients on the ramp surface.

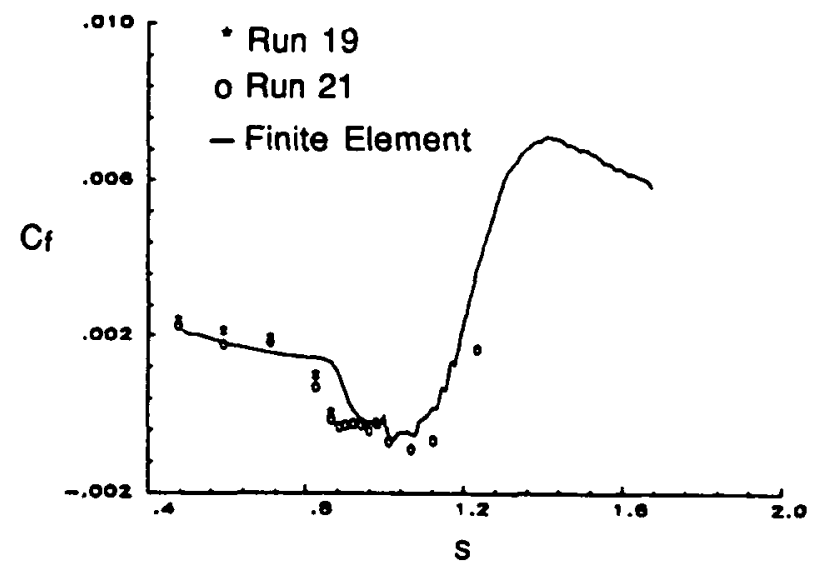

Fig. 34 Comparison of skin friction coefficient on ramp surface.

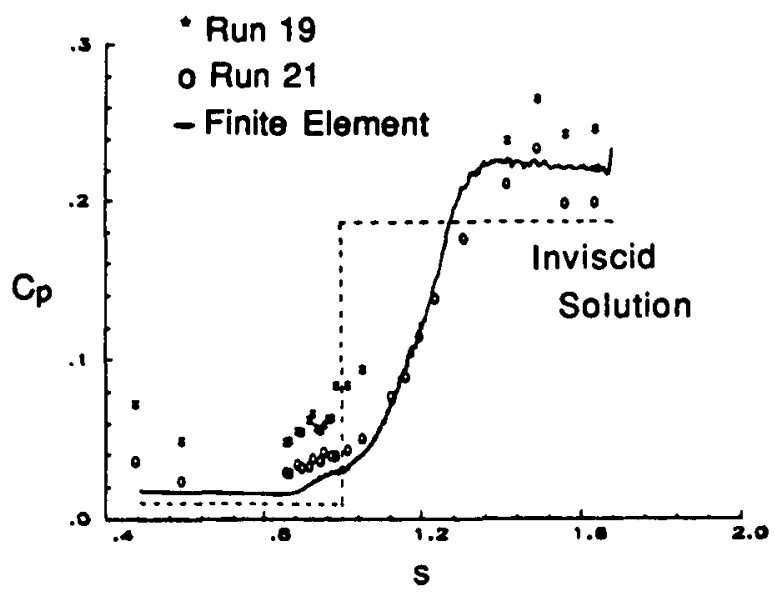

Fig. 35 Comparison of surface pressure coefficients on ramp surfaco.

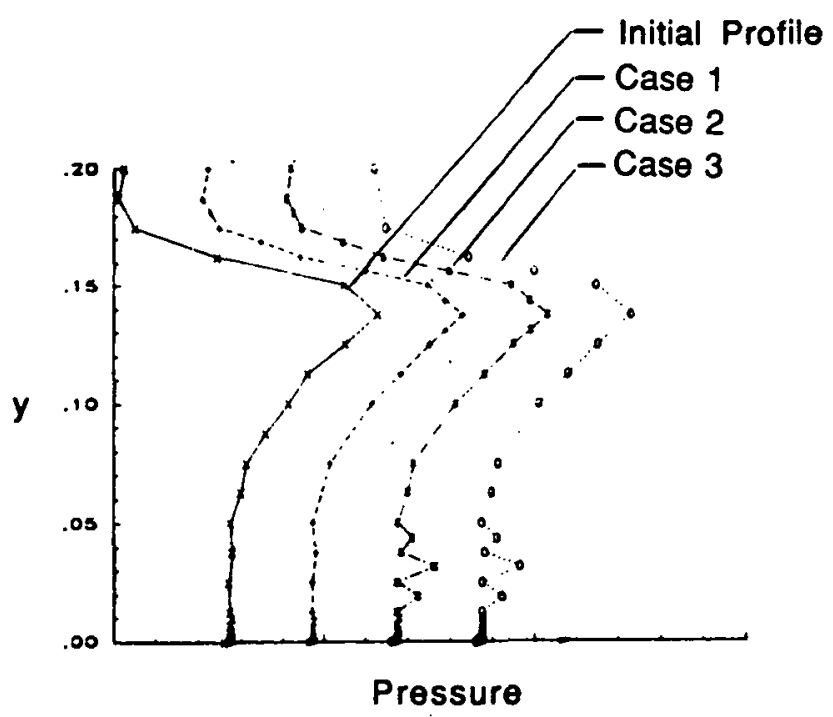

Fig. 36 Pressure profiles at location A showing effects of refinement. 


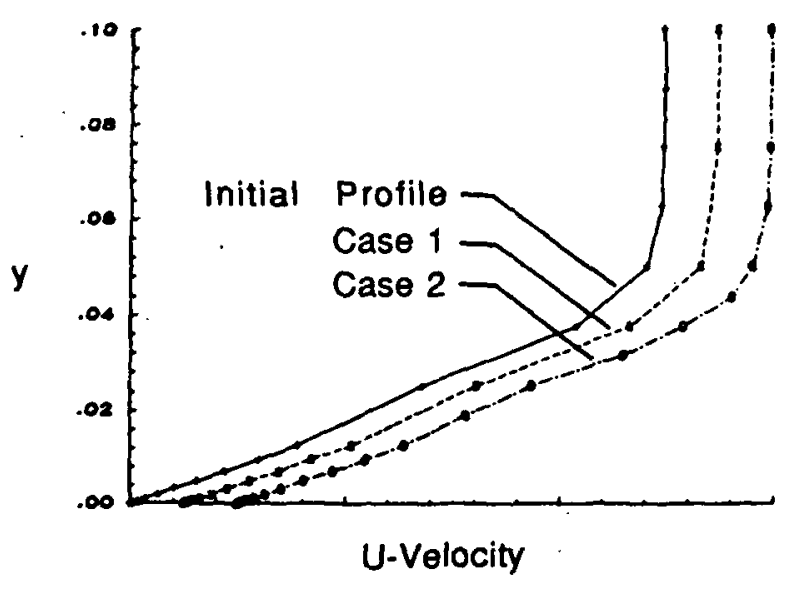

Fig. 37 Velocity profiles at location A on ramp.

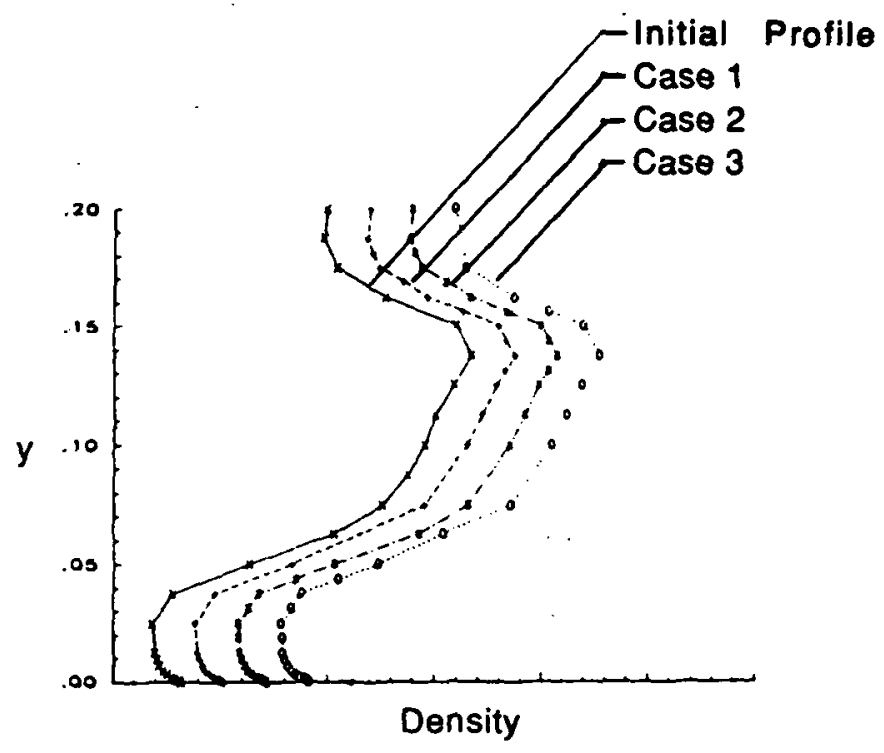

Fig. 38 Density profiles on ramp showing effects of refinement.

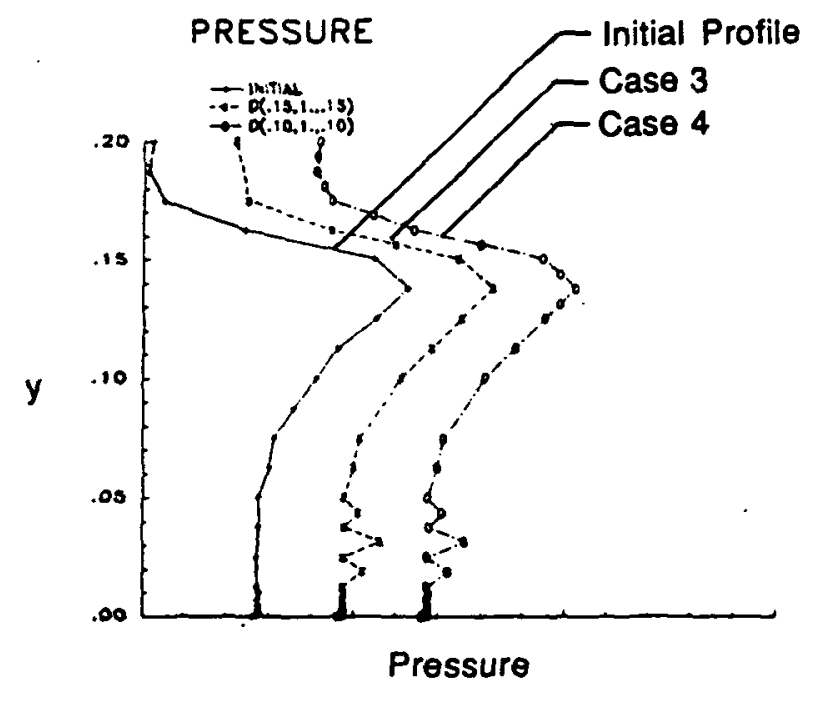

Fig. 39 Pressure profiles on ramp showing effects of varying threshold values.

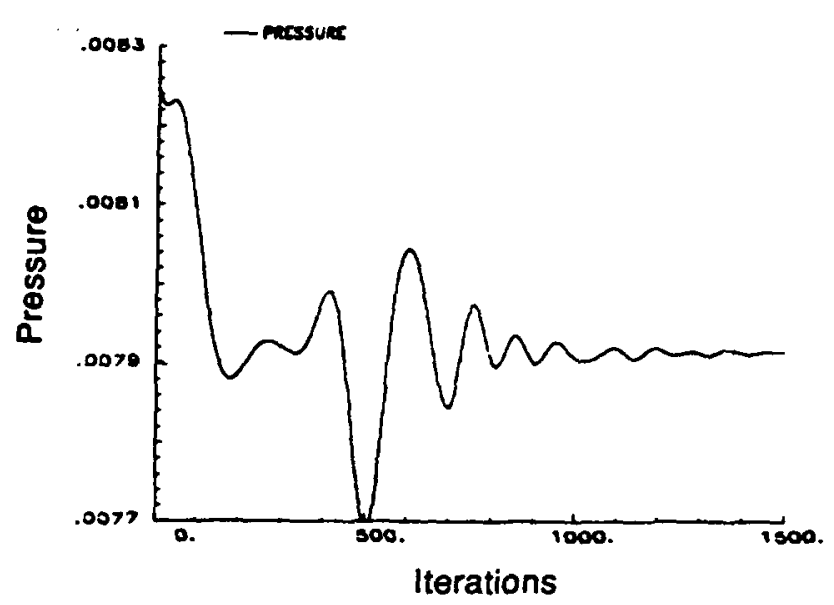

Fig. 40 Temporal history of pressure at typical new node within the boundary. 